\title{
Comparisons of Electromagnetic Scattering Properties of Real Hailstones and Spheroids
}

\author{
Zhiyuan Jiang, Matthew R. Kumjian, And Robert S. Schrom \\ Department of Meteorology and Atmospheric Science, The Pennsylvania State University, University Park, Pennsylvania
}

IAn Giammanco, TANya Brown-Giammanco, HeAther Estes, AND Ross Maiden

Insurance Institute for Business and Home Safety, Richburg, South Carolina

ANDREW J. HEYMSFIELD

National Center for Atmospheric Research, Boulder, Colorado

(Manuscript received 8 December 2017, in final form 8 November 2018)

\begin{abstract}
Severe $(>2.5 \mathrm{~cm})$ hail causes $>\$ 5$ billion in damage annually in the United States. However, radar sizing of hail remains challenging. Typically, spheroids are used to represent hailstones in radar forward operators and to inform radar hail-sizing algorithms. However, natural hailstones can have irregular shapes and lobes; these details significantly influence the hailstone's scattering properties. The high-resolution 3D structure of real hailstones was obtained using a laser scanner for hail collected during the 2016-17 Insurance Institute for Business and Home Safety (IBHS) Hail Field Study. Plaster casts of several record hailstones (e.g., Vivian, South Dakota, 2010) were also scanned. The S-band scattering properties of these hailstones were calculated with the discrete dipole approximation (DDA). For comparison, scattering properties of spheroidal approximations of each hailstone (with identical maximum and minimum dimensions and mass) were calculated with the T matrix. The polarimetric radar variables have errors when using spheroids, even for small hail. Spheroids generally have smaller variations in the polarimetric variables than the real hailstones. This increased variability is one reason why the correlation coefficient $\rho_{\mathrm{hv}}$ tends to be lower in observations than in forward-simulated cases using spheroids. Backscatter differential phase $\delta$ also is found to have large variance, particularly for large hailstones. Irregular hailstones with a thin liquid layer produce enhanced and more variable values for reflectivity factor at horizontal polarization $Z_{\mathrm{HH}}$, differential reflectivity $Z_{\mathrm{DR}}$, specific differential phase $K_{\mathrm{DP}}$, linear depolarization ratio (LDR), and $\delta$ compared with dry hailstones; $\rho_{\mathrm{hv}}$ is also significantly reduced.
\end{abstract}

\section{Introduction}

From 1949 to 2006, the estimated annual cost of damage caused solely by hail was $\$ 852$ million, and an average of 15 hail-related catastrophes (events causing $>\$ 1$ million in insured property losses) occurred each year (Chagnon et al. 2009). Further, some recent storms individually have caused over $\$ 1$ billion in insured property losses. For example, the recent 8 May 2017 hailstorm in Colorado caused $\$ 2.3$ billion in damages according to the Rocky Mountain Insurance Information Association, which makes it the state's record for the most damage caused by a single hailstorm. A hailstorm in San Antonio

Corresponding author: Zhiyuan Jiang, zxj113@psu.edu on 12 April 2016 caused $\$ 1.36$ billion in losses, making it the costliest hailstorm in Texas history (National Weather Service-San Antonio 2016).

Many studies have focused on detecting hailstorms with polarimetric radars. Reflectivity factor at horizontal polarization $Z_{\mathrm{HH}}$ and differential reflectivity $Z_{\mathrm{DR}}$ were first used for hail detection (e.g., Bringi et al. 1984; Leitao and Watson 1984; Aydin et al. 1986; Depue et al. 2007). Further, linear depolarization ratio (LDR; Bringi et al. 1986; Höller et al. 1994; Kennedy et al. 2001; Zeng et al. 2001), specific differential phase $K_{\mathrm{DP}}$ (Zrnić et al. 1988; Ryzhkov and Zrnić 1998), and correlation coefficient $\rho_{\text {hv }}$ (Balakrishnan and Zrnić 1990b; Picca and Ryzhkov 2012; Ryzhkov et al. 2013b) have been used in hail detection and algorithm development studies. 
Typically, small or negative $Z_{\mathrm{DR}}$, large LDR, and small $\rho_{\text {hv }}$ values collectively can indicate the presence of hailstones, based on observations (e.g., Hubbert et al. 1998; Picca and Ryzhkov 2012; Ortega et al. 2016) and theoretical scattering calculations (e.g., Aydin and Zhao 1990; Ryzhkov et al. 2013a). Large $K_{\mathrm{DP}}$ values have been tied to large concentrations of small melting hail (Kumjian and Lebo 2016; Kumjian et al. 2019).

According to the U.S. National Weather Service (NWS), severe $(>2.5 \mathrm{~cm})$ and significantly severe $(>5 \mathrm{~cm})$ hail is capable of inflicting substantial damage. Even though many algorithms have been developed for hail detection, determining hail size with weather radar observations remains a challenge (Blair et al. 2011; Kaltenboeck and Ryzhkov 2013; Kunkel et al. 2013; Ryzhkov et al. 2013b; Ortega et al. 2016). To better detect damaging hail in storms with radars, a better understanding of the scattering properties of hailstones and their signatures in radar observations is needed. In addition, hailstone scattering properties are also needed for radar forward operators for model evaluation and data assimilation.

For the purposes of scattering calculations, hailstones are usually assumed to have spheroidal shapes, and so the Rayleigh approximation (e.g., Jung et al. 2008) or the T-matrix method (e.g., Aydin and Zhao 1990; Balakrishnan and Zrnić 1990b; Depue et al. 2007; Ryzhkov et al. 2013a) are used. However, realistic hailstones exhibit various irregular shapes and can have protuberances or spikes known as lobes (e.g., Knight and Knight 1970b). These irregularities are thought to affect the scattering properties of hailstones. Balakrishnan and Zrnić (1990a) used simplified analytical calculations to demonstrate a reduction in $\rho_{\mathrm{hv}}$ for small particles with protuberances compared to similar-sized smooth particles. Using more detailed calculations, Mirković (2015) studied the effect of varying scales of surface roughness on scattering properties of artificial hailstones, which he modeled as spheroids. He found that, for severe and significantly severe hailstones, the roughness has a strong effect on the S-band scattering properties, specifically reducing $\rho_{\text {hv }}$ and increasing the backscatter differential phase shift $\delta$. Therefore, the smooth spheroidal shapes used in many studies and that serve as the basis for forward operators (e.g., Jung et al. 2008; Ryzhkov et al. 2011, 2013a; Kumjian et al. 2014) do not fully capture the scattering properties of hailstones. To date, however, attempts to account for the irregularities of natural hailstones have used highly idealized particles such as spheres or spheroids with uniform roughness/ spikes applied (Balakrishnan and Zrnić 1990a; Mirković 2015). The impact of nonspheroidal particles and/or those with more natural protuberances over a range of sizes on scattering remains to be quantified.
Recently, the Insurance Institute for Business and Home Safety (IBHS) hail measurement field project obtained measurements of real hailstones, including detailed scans of their three-dimensional structures (Giammanco et al. 2017). These three-dimensional structures can be used to calculate the scattering properties of real hailstones. In this study, we calculate the scattering properties and polarimetric variables of real hailstones and compare the results to spheroidal particles often used to approximate them. To the extent of our knowledge, this is the first time that real hailstone structures are used for scattering calculations.

This paper is structured as follows. Section 2 introduces the methods used for the scattering calculations and the formulas for the polarimetric radar variables. Section 3 shows the results comparing real hailstones and spheroids in terms of backscattering properties of different incident directions and polarimetric radar variables with orientation distribution assumptions. Given that hailstones below the melting layer usually are wet, a comparison between dry and wet real hailstones is also given in section 3 . In the end, a summary of the current study and discussion are given in section 4 .

\section{Methods}

The emergence of 3D laser scanning technology has allowed for new applications to be explored. Giammanco et al. (2017) used a handheld threedimensional scanner system (Fig. 1) to successfully create high-resolution digital models of natural hailstones. During the 2016 and 2017 IBHS field campaigns, over 100 digital models of natural hailstones were obtained. Each hailstone was photographed, weighed, and measured with a caliper prior to scanning. For scanning, the target stone is placed on a mount, allowing the operator to rotate the hailstone to complete the scan and minimize movement within the field research vehicle. Hailstones were scanned at a resolution of $0.008 \mathrm{~cm}$; the system had a measurement error of $\pm 0.004 \mathrm{~cm}$. Scans took approximately 2-3 min to complete. For more detailed information on the scanner system, data collection, and data processing, see Giammanco et al. (2017). For this study, we use eight hailstones scanned by this system (Fig. 2; Table 1). These include scans of plaster casts of three U.S. record hailstones: Coffeyville, Kansas (1970; hailstone 7); Aurora, Nebraska (2003; hailstone 6); and Vivian, South Dakota, (2010; hailstone 8) supplied by NCAR.

The scattering calculations are performed using the discrete dipole approximation (DDA). DDA, first developed by DeVoe (1964), is a method to calculate 


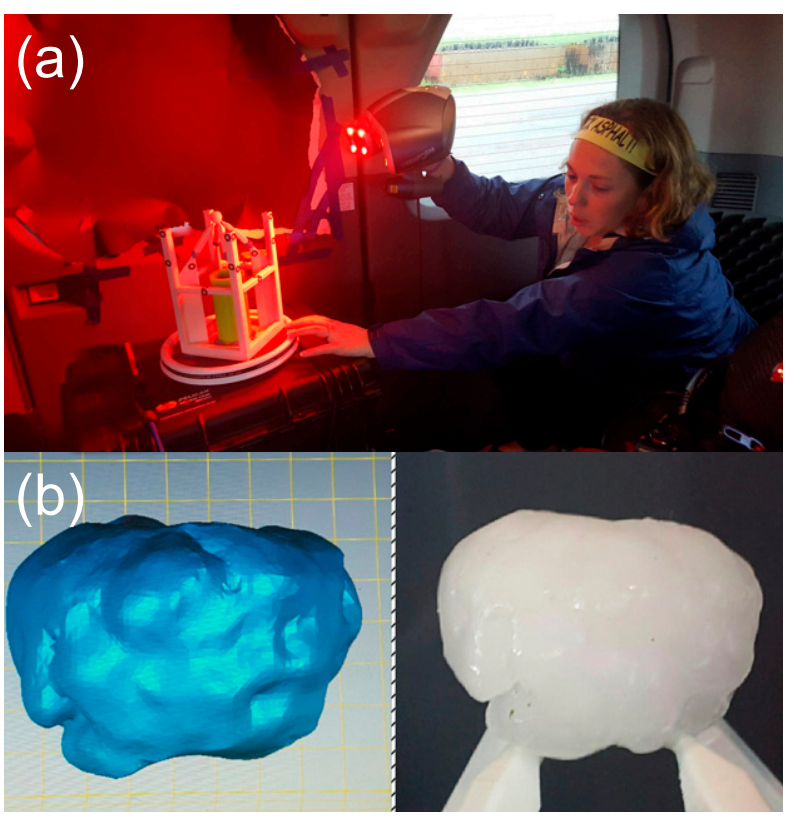

FIG. 1. (a) IBHS scientist Heather Estes scanning a hailstone with the three-dimensional scanning system. The hailstone is on top of the mount (white structure). (b) (left) Example of the rendered three-dimensional surface and (right) a photograph of the actual hailstone on the mount.

the electromagnetic scattering properties of arbitrary shapes. We use the freely available Amsterdam DDA (ADDA) code (Yurkin and Hoekstra 2011) in this study. The three-dimensional hailstone surfaces obtained from the scanner are used to construct the particles for the DDA calculations: the volume created by the hailstone surface is filled with dipoles. Draine and Flatau (1994) suggest that dipole size should be smaller than $\lambda / 4 \pi|n|$, where $n$ is the particle refractive index and $\lambda$ is the wavelength of the illuminating radiation. Yurkin and Hoekstra (2011) also suggest the same rule and point out that the dipole size should be much smaller than both $\lambda$ and the particle's minimum dimension $D_{\min }$. Here, we set the dipole size to $\max \left(D_{x}, D_{y}, D_{z}\right) / 100$, where $D_{x}, D_{y}$, and $D_{z}$ represent the dimensions in the $x, y$, and $z$ axes, respectively. Because the dimensions $D_{x}, D_{y}$, and $D_{z}$ have roughly the same order of magnitude, the requirement for the dipole size to be smaller than the particle's minimum dimension is satisfied. The largest dipole size in the set of hailstones is $1.51 \mathrm{~mm}$, which is nearly two orders of magnitude smaller than the S-band radar wavelength at which we perform the calculations. During 3D scanning, liquid water on the surface of hailstones is removed (Giammanco et al. 2017). The hailstones are assumed to have the relative permittivity of solid ice.

Because spheroids are widely used in computations of hydrometeor scattering properties, including for hailstones (as described above), we also perform scattering calculations for equivalent spheroids for each hailstone in the dataset. Commonly, the size of hailstones is used in radar forward simulators to constrain the spheroids. As such, we prescribe that the equivalent spheroids have identical maximum and minimum dimensions as the real hailstones. However, keeping the density equal to that of solid ice would result in the spheroids having excessive mass relative to the real hailstones, thereby producing an inconsistency within the computed radar variables affected by mass (i.e., $Z_{H}$ and $\left.K_{\mathrm{DP}}\right)$. To mitigate this issue, we reduced the density of the spheroids such that the particle mass is equivalent to the real hailstone mass. The T-matrix code from Mishchenko (2000) is used for these scattering calculations. Tests of spheroid calculations using the T-matrix and DDA codes revealed negligible differences (not shown). Thus, any differences in the scattering properties between spheroids and the real hailstones are related to differences in the particle shapes, not the calculation methods.

To calculate the amplitude scattering matrix and thus the dual-polarization radar variables for the hailstones, we need their orientation relative to the direction and polarization of the incident electromagnetic wave. We assume that the particles are oriented such that their minimum dimension is aligned along the $z$ axis and the maximum dimension in the horizontal plane (Fig. 3). The direction of the incident radiation is specified by its elevation angle $\theta$ and azimuth angle $\varphi$. For the real hailstones, we select elevation angles from $-90^{\circ}$ to $90^{\circ}$ in $10^{\circ}$ intervals, and azimuth angles from $0^{\circ}$ to $340^{\circ}$ with $20^{\circ}$ intervals. Because the real hailstones can be highly irregular in shape, varying the elevation and azimuthal angles allows for assessing the variability of the hailstone scattering properties as a function of the incident radiation direction and/or the hailstone orientation. Because oblate spheroids are symmetric about their minor dimension, their scattering properties depend only on elevation angle. Thus, variability of the natural hailstones' scattering properties can be seen as a measure of the uncertainty in their scattering properties. Because such uncertainty is not accounted for in most existing forward operators and calculations for hailstones, this can be seen as a novel aspect of the current study and will be discussed further in section 3 .

The polarization direction is important in polarimetric radar observations, and is the key to develop all the polarimetric radar variables. We define the horizontal ("h") polarization direction to be in the direction of $\hat{e}_{\varphi}$, which is always in the $x-y$ plane (Fig. 3). Therefore, the vertical ("v") polarization direction is determined by the h-polarization direction and the propagation direction 


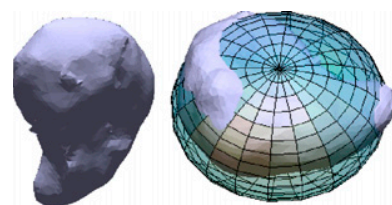

1

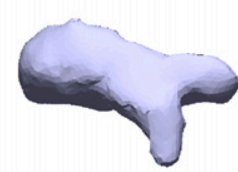

4
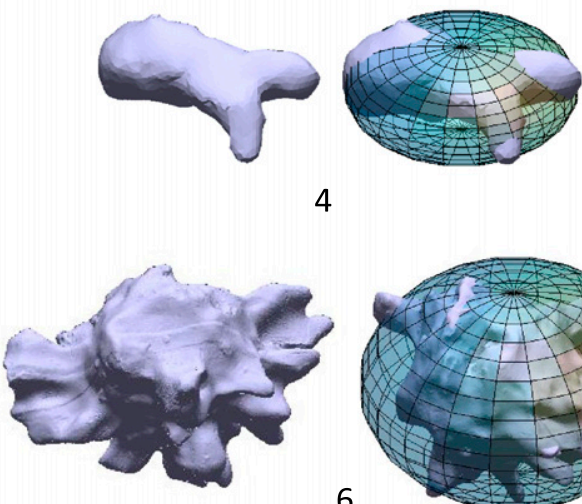

6
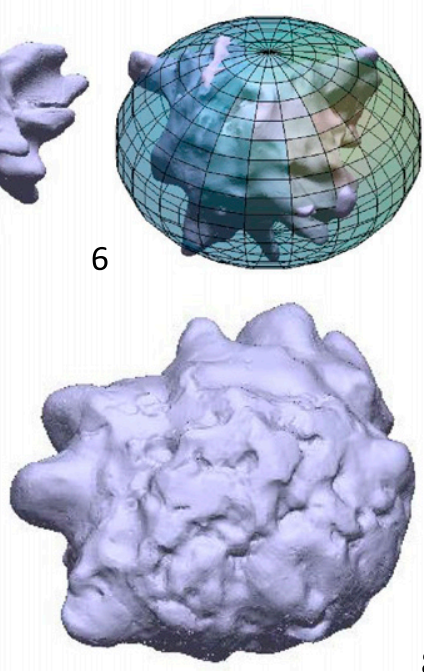

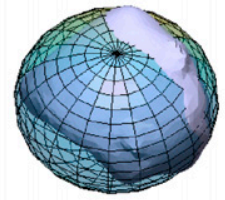

2

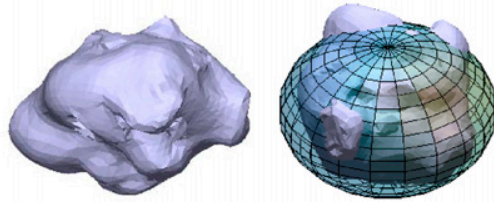

3

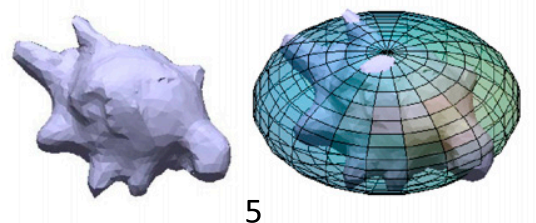

5

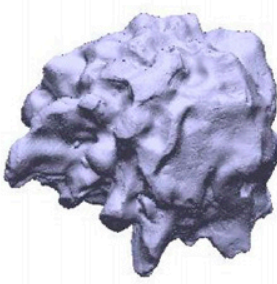

7
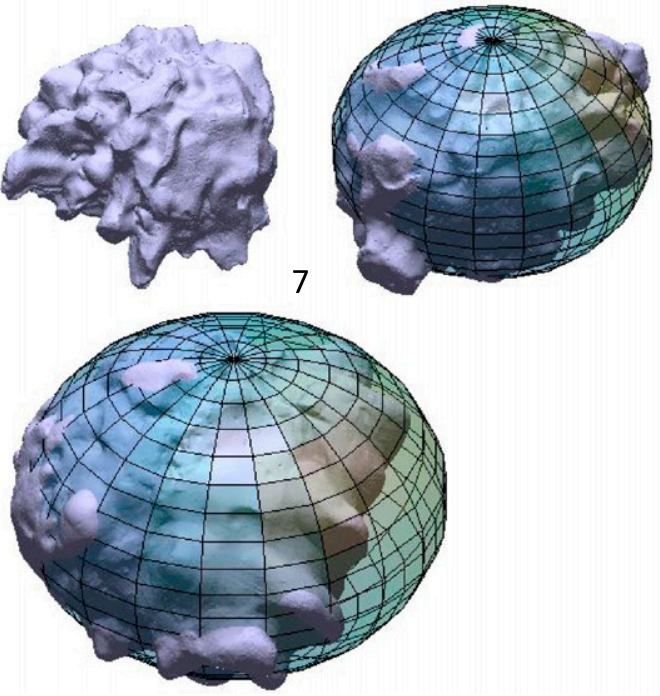

FIG. 2. Digital 3D renderings of the hailstones used in this study and the spheroids with the same maximum and minimum dimensions as the corresponding hailstones. Not to scale.

$\hat{k}$ of the incident wave using the right-hand rule ( $\mathrm{Lu}$ et al. 2016), such that $\hat{k}=\hat{e}_{\mathrm{h}} \times \hat{e}_{\mathrm{v}}$ (Fig. 3). The advantage of using the aforementioned orientation setup is that rotating particles is equivalent to changing the elevation and azimuthal angles of the incident radiation direction. Changing the azimuth angle $\varphi$ is equivalent to rotating the particle around the $z$ axis, and changing the elevation angle $\theta$ is equivalent to rotating the particle about the h-polarization direction $\hat{e}_{\varphi}$. The third degree of freedom is that the particle can also cant (i.e., rotate) about the propagation direction of incident radiation. We account for this canting by considering rotations of the $\mathrm{h}$ - and v-polarization directions about an angle $\alpha$ (Fig. 4). The relationship between incident and scattered electric field of a plane wave is related by the scattering amplitude matrix $\mathbf{S}$ before canting and by $\mathbf{S}^{\prime}$ after canting:

$$
\left(\begin{array}{c}
E_{\mathrm{h}}^{s} \\
E_{\mathrm{v}}^{s}
\end{array}\right)=\frac{e^{-i k r}}{r} \mathbf{S}\left(\begin{array}{c}
E_{\mathrm{h}}^{i} \\
E_{\mathrm{v}}^{i}
\end{array}\right)
$$

and

$$
\left(\begin{array}{c}
E_{\mathrm{h}}^{\prime s} \\
E_{\mathrm{v}}^{\prime s}
\end{array}\right)=\frac{e^{-i k r}}{r} \mathbf{S}^{\prime}\left(\begin{array}{c}
E_{\mathrm{h}}^{\prime i} \\
E_{\mathrm{v}}^{\prime i}
\end{array}\right)
$$

where

$$
\mathbf{S}=\left(\begin{array}{ll}
S_{\mathrm{hh}} & S_{\mathrm{vh}} \\
S_{\mathrm{hv}} & S_{\mathrm{vv}}
\end{array}\right)
$$

and

$$
\mathbf{S}^{\prime}=\left(\begin{array}{cc}
S_{\mathrm{hh}}^{\prime} & S_{\mathrm{vh}}^{\prime} \\
S_{\mathrm{hv}}^{\prime} & S_{\mathrm{vv}}^{\prime}
\end{array}\right)
$$

TABLE 1 . The maximum and minimum dimensions $(\mathrm{cm})$ of each hailstone.

\begin{tabular}{lcccccrrr}
\hline & \multicolumn{8}{c}{ Particle No. } \\
\cline { 2 - 9 } & 1 & 2 & 3 & 4 & 5 & \multicolumn{1}{c}{6} & \multicolumn{1}{c}{7} & \multicolumn{1}{c}{8} \\
\hline Max & 3.60 & 2.90 & 3.27 & 3.91 & 3.70 & 12.01 & 10.44 & 17.04 \\
Min & 2.09 & 2.15 & 2.21 & 1.60 & 1.70 & 8.59 & 6.61 & 11.70 \\
\hline
\end{tabular}




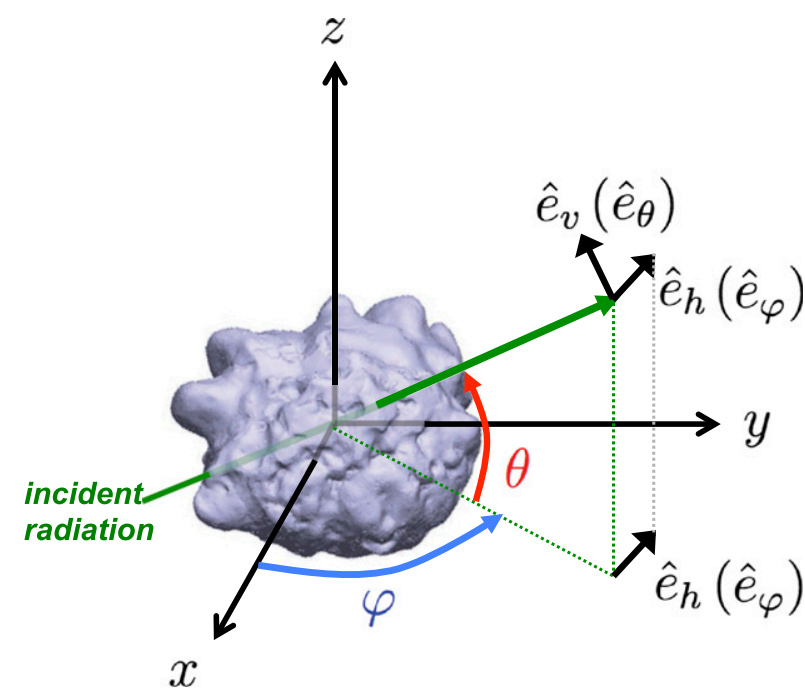

FIG. 3. Schematic indicating the orientation of hailstones and directions of incident radiation and polarization. Hailstones are oriented with their minimum dimension along the $z$ axis. Incident radiation (green arrow) is specified by elevation angle $\theta$ (red) and azimuth angle $\varphi$ (blue). The horizontal polarization direction $\hat{e}_{\mathrm{h}}$ is the same as unit vector $\hat{e}_{\varphi}$ and vertical polarization direction $\hat{e}_{\mathrm{v}}$ is the same as unit vector of $\hat{e}_{\theta}$.

and superscripts $i$ and $s$ indicate incident and scattered radiation, respectively. Canting the particle clockwise with angle $\alpha$ is equivalent to rotating the h- and v-polarization directions anticlockwise by angle $\alpha$ (Fig. 4). The incident and scattered electric field before and after canting are related as follows:

$$
\left(\begin{array}{c}
E_{\mathrm{h}}^{\prime s, i} \\
E_{\mathrm{v}}^{\prime s, i}
\end{array}\right)=\left(\begin{array}{cc}
\cos \alpha & \sin \alpha \\
-\sin \alpha & \cos \alpha
\end{array}\right)\left(\begin{array}{c}
E_{\mathrm{h}}^{s, i} \\
E_{\mathrm{v}}^{s, i}
\end{array}\right) .
$$

Thus, the relationship between the scattering amplitude matrix $\mathbf{S}$ before canting and $\mathbf{S}^{\prime}$ after canting is

$$
\begin{aligned}
\left(\begin{array}{cc}
S_{\mathrm{hh}}^{\prime} & S_{\mathrm{vh}}^{\prime} \\
S_{\mathrm{hv}}^{\prime} & S_{\mathrm{vv}}^{\prime}
\end{array}\right)= & \left(\begin{array}{cc}
\cos \alpha & \sin \alpha \\
-\sin \alpha & \cos \alpha
\end{array}\right)\left(\begin{array}{cc}
S_{\mathrm{hh}} & S_{\mathrm{vh}} \\
S_{\mathrm{hv}} & S_{\mathrm{vv}}
\end{array}\right) \\
& \times\left(\begin{array}{cc}
\cos \alpha & -\sin \alpha \\
\sin \alpha & \cos \alpha
\end{array}\right) .
\end{aligned}
$$

Note that the forward scattering amplitude matrix $\mathbf{S}_{\mathrm{FSA}}^{f}$ following the forward scatter alignment (FSA) convention is needed for the $K_{\mathrm{DP}}$ calculation, whereas the backward scattering amplitude matrix $\mathbf{S}_{\mathrm{BSA}}^{b}$ following the backscatter alignment (BSA) convention is needed for the $Z_{\mathrm{HH}}, Z_{\mathrm{DR}}, \rho_{\mathrm{hv}}, \mathrm{LDR}$, and $\delta$ calculations. Even though the scattering amplitude matrices following FSA and BSA conventions are related, as in Bringi and Chandrasekar (2001),

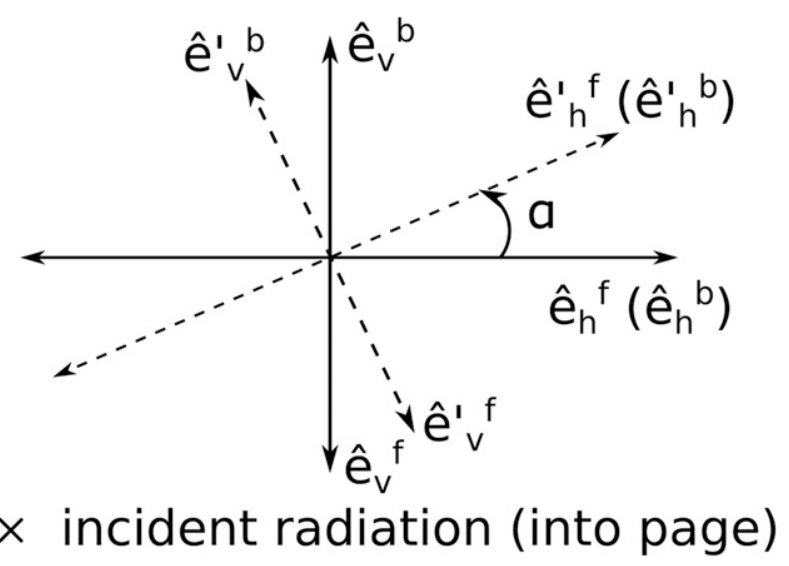

FIG. 4. Schematic showing the amplitude scattering matrix converted by canting around the incident radiation direction. The polarization directions are solid lines before canting and dashed lines after canting by angle $\alpha$. The horizontal and vertical polarization is indicated by the subscripts $\mathrm{h}$ and $\mathrm{v}$, respectively. The superscripts $f$ and $b$ indicate forward and backward scattering, respectively.

$$
\mathbf{S}_{\mathrm{BSA}}=\left(\begin{array}{cc}
-1 & 0 \\
0 & 1
\end{array}\right) \mathbf{S}_{\mathrm{FSA}},
$$

they need to be in the correct convention before applying Eq. (6) to account for canting. The first set of calculations thus correspond to single particle scattering at different incident angles, as described above.

In the next set of calculations, the polarimetric radar variables are calculated using the amplitude scattering matrix with additional assumptions about the hailstone orientation and canting angle distributions. For real hailstones, $\varphi$ is assumed to be uniformly distributed (i.e., there is no preferred azimuthal orientation for the hailstones). We assume $\theta$ and $\alpha$ follow the same Gaussian distribution with $0^{\circ}$ mean and standard deviation $\sigma$, which represents how much the particles wobble in free fall. In the literature, $\sigma$ is usually set to be $10^{\circ}$ or less for pristine ice crystals and raindrops, which tend to be more stable during free fall, and up to $40^{\circ}$ or larger for aggregates and hailstones that tend to tumble more chaotically (e.g., Jung et al. 2008; Ryzhkov et al. 2011, 2013a; Jiang et al. 2017). Here we compute the radar variables for $\sigma$ values ranging from $10^{\circ}$ to $80^{\circ}$, as well as for a random distribution of canting angles.

Six polarimetric radar variables are calculated in this study: $Z_{\mathrm{HH}}, Z_{\mathrm{DR}}, K_{\mathrm{DP}}, \rho_{\mathrm{hv}}, \mathrm{LDR}$, and $\delta$. Owing to the limited number of hailstones considered here, the main focus of this study is on each hailstone and the comparison with its spheroidal approximation. Therefore, the particle size distribution (PSD) is assumed to be monodisperse, and integration over the size distribution is simply replaced by multiplying by the number concentration $N$. For the spheroids, the integration over 
$\varphi$ is further eliminated owing to symmetry. This is a primary limitation of the approach herein; as more hailstones are added to the 3D scanner database in the future, there may be enough to cover a broad range of sizes to be able to test realistic PSDs.

The formulas to calculate the polarimetric variables from Aydin and Zhao (1990) are modified as follows:

$$
\begin{aligned}
Z_{\mathrm{HH}}\left[\mathrm{mm}^{-6} \mathrm{~m}^{-3}\right]= & \frac{N \lambda^{4}}{\pi^{5}\left|K_{w}\right|^{2}} \iiint 4 \pi\left|S_{\mathrm{hh}}^{b}(\alpha, \theta, \varphi)\right|^{2} \\
& \times p(\alpha) p(\theta) p(\varphi) d \alpha d \theta d \varphi \\
Z_{\mathrm{DR}}[\mathrm{dB}]= & 10 \log _{10}\left(\frac{Z_{\mathrm{hh}}}{Z_{\mathrm{vv}}}\right)
\end{aligned}
$$

$$
\begin{aligned}
\rho_{\mathrm{hv}} & =\frac{\iiint S_{\mathrm{vv}}^{b}(\alpha, \theta, \varphi) S_{\mathrm{hh}}^{* b}(\alpha, \theta, \varphi) p(\alpha) p(\theta) p(\varphi) d \alpha d \theta d \varphi}{\left[\iiint\left|S_{\mathrm{vv}}^{b}(\alpha, \theta, \varphi)\right|^{2} p(\alpha) p(\theta) p(\varphi) d \alpha d \theta d \varphi \iiint\left|S_{\mathrm{hh}}^{b}(\alpha, \theta, \varphi)\right|^{2} p(\alpha) p(\theta) p(\varphi) d \alpha d \theta d \varphi\right]^{1 / 2}}, \\
K_{\mathrm{DP}}\left[{ }^{\circ} \mathrm{km}^{-1}\right] & =N \frac{180}{\pi}\left(10^{-3}\right) \frac{2 \pi}{k} \operatorname{Re}\left\{\iiint\left[S_{\mathrm{hh}}^{f}(\alpha, \theta, \varphi)-S_{\mathrm{vv}}^{f}(\alpha, \theta, \varphi)\right] p(\alpha) p(\theta) p(\varphi) d \alpha d \theta d \varphi\right\}, \\
\operatorname{LDR}[\mathrm{db}] & =10 \log _{10}\left[\iiint\left|S_{\mathrm{hv}}^{b}(\alpha, \theta, \varphi)\right|^{2} p(\alpha) p(\theta) p(\varphi) d \alpha d \theta d \varphi\right], \quad \text { and }
\end{aligned}
$$

with elevation angle, similar to that of the spheroids

$$
\delta[\operatorname{rad}]=\arg \left(\rho_{\mathrm{hv}}\right),
$$

where $N$ is the number concentration $\left(\mathrm{m}^{-3}\right), \lambda$ is the wavelength (mm), $\left|K_{w}\right|^{2}$ is the dielectric factor for liquid water $(\sim 0.93$ at $\mathrm{S}$ band), and $k$ is the wavenumber $\left(\mathrm{mm}^{-1}\right)$. The scattering amplitude matrix $\mathbf{S}$ has units of millimeters, and the superscripts $b$ and $f$ represent backscattering and forward scattering, respectively. The asterisk represents the complex conjugate. The $p(\alpha)$, $p(\theta)$, and $p(\varphi)$ terms represent the distributions of canting, elevation, and azimuth angles, respectively. All three angles $(\alpha, \theta, \varphi)$ are included in the integration as to fully consider the variability of particle orientation, which is critical because $\rho_{\text {hv }}$ strongly depends on the scattering variability over different orientations (e.g., Jameson and Mueller 1985; Balakrishnan and Zrnić 1990a; Doviak and Zrnić 1993; Bringi and Chandrasekar 2001; Kumjian 2013).

\section{Results}

\section{a. Scattering properties of single particles as a function of orientation}

The single scattering properties of hailstones as a function of incident radiation direction are plotted in Figs. 5 and 6 , which also show a comparison with spheroids. The number concentration $N$ is fixed at $1 \mathrm{~m}^{-3}$ for each. For hailstones with sizes around 3-4 cm (hailstones $1-5), Z_{\mathrm{HH}}, Z_{\mathrm{DR}}$, and $K_{\mathrm{DP}}$ follow a similar pattern
(Fig. 5). $Z_{\mathrm{HH}}$ decreases with increasing incident elevation angle between $-90^{\circ}$ and $0^{\circ}$, and increases for elevation angles $>0^{\circ}$. Note that, in our framework, $\pm 90^{\circ}$ incident elevation angles correspond to the direction of wave propagation along the particle's minor axis. In contrast, both $Z_{\mathrm{DR}}$ and $K_{\mathrm{DP}}$ increase with increasing incident elevation angles between $-90^{\circ}$ and $0^{\circ}$ and then decrease for elevation angles $>0^{\circ}$. For hailstones of sizes comparable to or larger than the wavelength ( $\geq 10 \mathrm{~cm}$; hailstones 6-8), all the polarimetric variables fluctuate substantially with varying incident direction (Fig. 6); no well-behaved pattern is observed. Based on these size and scattering characteristics, the hailstones are divided into 4 groups that are described below.

Hailstones 1-3 have relatively smooth surfaces, lacking lobes and protuberances. For these hailstones, $Z_{\mathrm{HH}}$ is about the same as the corresponding spheroids. However, for a given elevation angle, the real hailstones exhibit $1-3 \mathrm{~dB}$ variability in $Z_{\mathrm{HH}}$ across the different azimuthal angles. Such a range in values could provide an estimate of the natural variability in scattering properties for particles of that size and mass. The median $Z_{\mathrm{DR}}$ for the real hailstones is within the same range as for the corresponding spheroids, although the real hailstones exhibit 1-2 dB of variability with azimuthal angle not captured by the spheroids. Similar to $Z_{\mathrm{DR}}, K_{\mathrm{DP}}$ of the real hailstones and spheroids is also consistent, though the real hailstones 

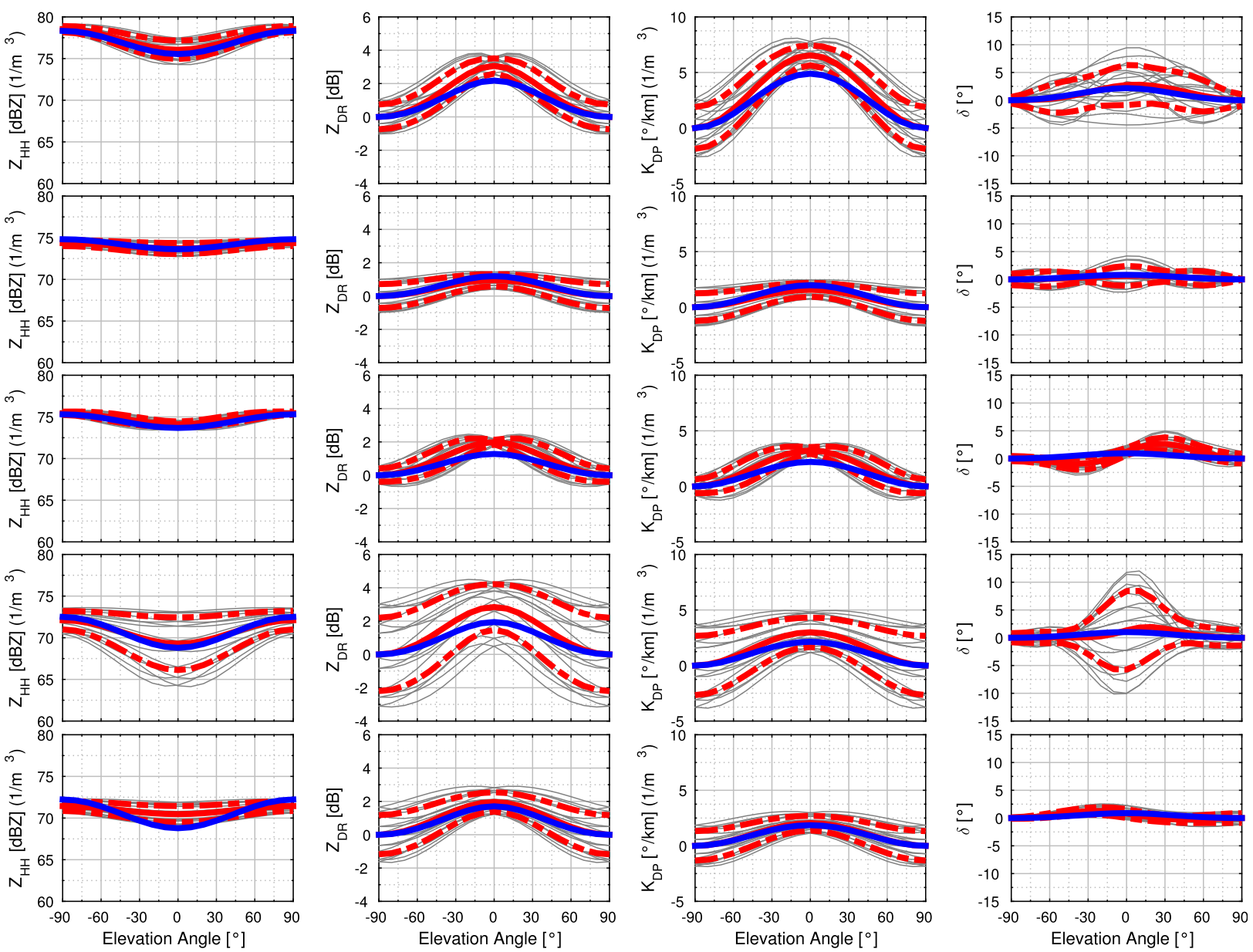

FIG. 5. Plots of (left to right) $Z_{\mathrm{HH}}, Z_{\mathrm{DR}}, K_{\mathrm{DP}}$, and $\delta$ as a function of incident elevation angle for (top to bottom) hailstones 1-5. The number concentration is assumed to be $1 \mathrm{~m}^{-3}$ and the canting angle is $0^{\circ}$. For hailstones, each gray line represents one azimuth angle. The red solid line represents the average value over all azimuth angles, while the red dashed lines show plus and minus one standard deviation. The blue line shows the results for spheroids.

have variations as large as $5^{\circ} \mathrm{km}^{-1}$ for different azimuthal angles. The value of $\delta$ fluctuates across the different incident directions with both positive and negative values for the real hailstones, but with only very small positive values for the spheroids. Considering the variability in elevation and azimuthal angles, the overall range of variability of $Z_{\mathrm{HH}}$ is about the same between the hailstones and spheroids, whereas the real hailstones have a slightly larger variability in $Z_{\mathrm{DR}}, K_{\mathrm{DP}}$, and $\delta$.

Hailstones 4 and 5 have similar sizes and bounding ellipsoidal shape (similar $a, b$, and $c$ axes), and both are small compared to the radar wavelength. However, these hailstones have highly irregular shapes: hailstone 4 resembles a fish or "rabbit head," whereas the shape of hailstone 5 is like a "spiky football." Despite their relatively small sizes (about $\sim 1 / 3$ of the wavelength), we are interested in how these irregular shapes affect the radar variables. Overall, $Z_{\mathrm{HH}}, Z_{\mathrm{DR}}$, $K_{\mathrm{DP}}$, and $\delta$ of the spheroids are within the range of the real hailstones, but the real hailstones exhibit larger variability, as expected. However, it is surprising to find that the variability among incident azimuth angles is much greater for the relatively smooth hailstone 4 (rabbit head) than hailstone 5 (spiky football). Hailstone 4 has variations (i.e., ranges of values over different elevation and azimuthal angles) of $10 \mathrm{~dB}$, $8 \mathrm{~dB}, 7^{\circ} \mathrm{km}^{-1}$, and $20^{\circ}$ for $Z_{\mathrm{HH}}, Z_{\mathrm{DR}}, K_{\mathrm{DP}}$, and $\delta$, respectively, whereas the variation of hailstone 5 is only about $3 \mathrm{~dB}, 4.5 \mathrm{~dB}, 4.5^{\circ} \mathrm{km}^{-1}$, and $4^{\circ}$ for $Z_{\mathrm{HH}}, Z_{\mathrm{DR}}$, $K_{\mathrm{DP}}$, and $\delta$, respectively. Thus, despite the "spiky" appearance of hailstone 5, the protuberances are evidently not large enough and/or are too uniform to produce substantial variability in its scattering properties. In contrast, hailstone 4 (rabbit head) has a center of mass displaced from its geometric center, leading to 

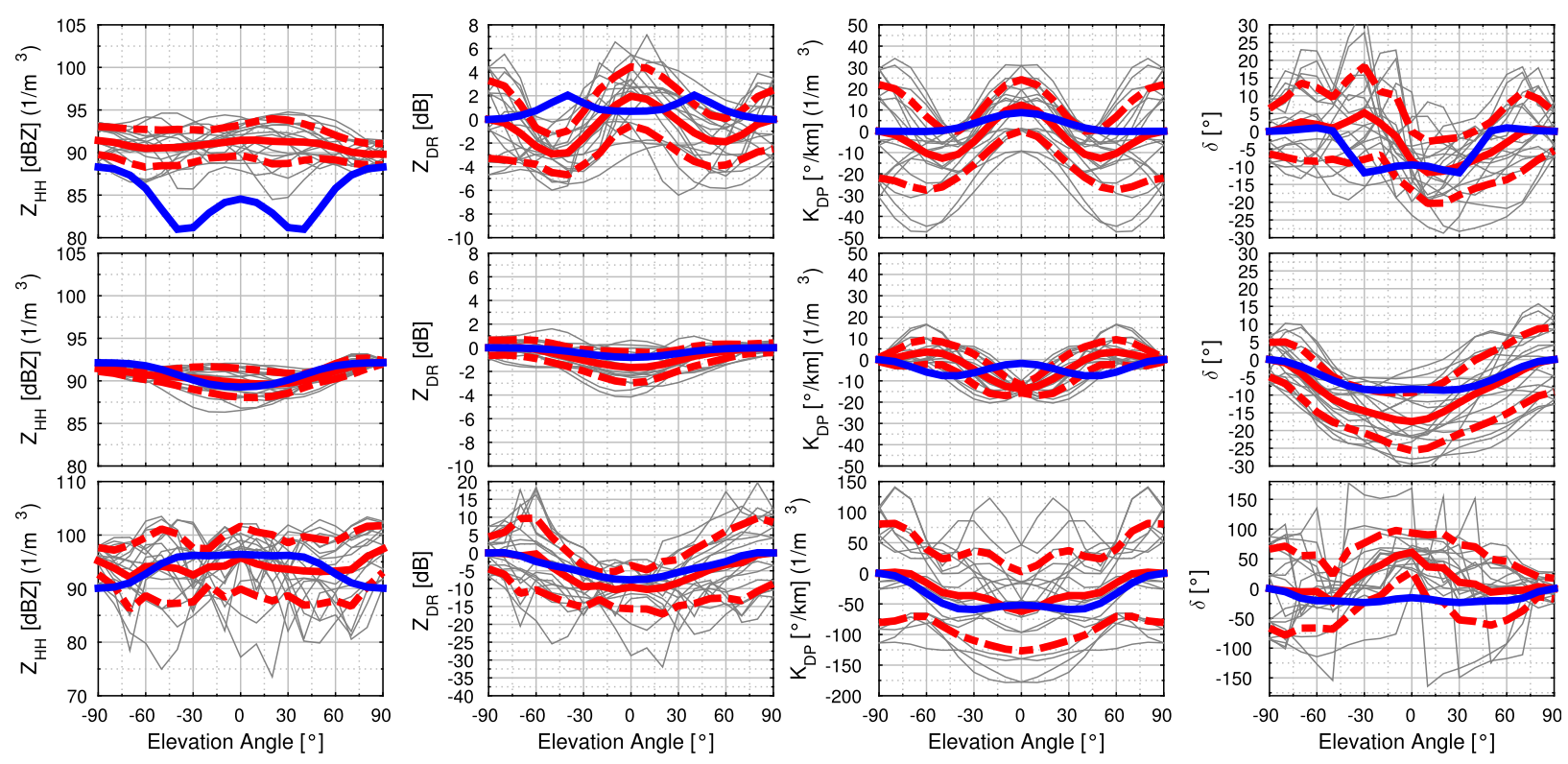

FIG. 6. As in Fig. 5, but for hailstones 6-8. Note the ranges of the ordinate axes change for hailstone 8.

greater variability in its scattering properties as it is rotated through different orientation angles.

In contrast to these small particles, hailstones 6 and 7 have sizes comparable to the S-band wavelength $(\sim 10 \mathrm{~cm})$. The fluctuation of each variable is large and unpredictable. Hailstone 6 has about $10 \mathrm{~dB}$ variation in $Z_{\mathrm{HH}}, 11 \mathrm{~dB}$ in $Z_{\mathrm{DR}}, 78^{\circ} \mathrm{km}^{-1}$ in $K_{\mathrm{DP}}$, and about $64^{\circ}$ in $\delta$. Hailstone 7 variations in $Z_{\mathrm{HH}}, Z_{\mathrm{DR}}, K_{\mathrm{DP}}$, and $\delta$ are about $6 \mathrm{~dB}, 6 \mathrm{~dB}, 37^{\circ} \mathrm{km}^{-1}$, and $48^{\circ}$, respectively. Because of these hailstones' irregular shapes, the patterns are strongly asymmetric about elevation angle or azimuthal angle. The variation with elevation angle for the associated spheroids also becomes large, especially for $K_{\mathrm{DP}}$ and $\delta$. The median $Z_{\mathrm{HH}}$ of hailstone 6 is close to the maximum in spheroid $Z_{\mathrm{HH}}$ for elevation angles $-90^{\circ}$ and $90^{\circ}$, whereas there is about a 5-10-dB difference for other angles. $Z_{\mathrm{DR}}$ and $K_{\mathrm{DP}}$ can be strongly positive or negative for hailstone $6 . Z_{\mathrm{HH}}$ differences between hailstone 7 and its matching spheroid are smaller, and $Z_{\mathrm{DR}}$, $K_{\mathrm{DP}}$, and $\delta$ are mainly negative.

Hailstone 8 (the record Vivian, South Dakota, hailstone) is much larger than the other hailstones and is larger than the radar wavelength. Its scattering properties are also different from the other large stones: each polarimetric variable fluctuates wildly, with a range of fluctuation much larger than that of the spheroid's fluctuations. The value of $Z_{\mathrm{HH}}$ varies by nearly $30 \mathrm{~dB}$, with some orientation angles producing $Z_{\mathrm{HH}}$ comparable to or less than $Z_{\mathrm{HH}}$ of the smallest hailstones in the dataset (cf. hailstones 2 and 5). This implies that, for equal number concentration of hailstones, $Z_{\mathrm{HH}}$ may be of limited utility for estimating hail size. The value of $Z_{\mathrm{DR}}$ varies by over $50 \mathrm{~dB}$, though median values are mainly negative. The value of $K_{\mathrm{DP}}$ varies by $318^{\circ} \mathrm{km}^{-1}$, with negative median values but enormous spread. Finally, $\delta$ fluctuates wildly, covering nearly the entire range $\pm 180^{\circ}$. Note that such variation only represents the scattering properties of a single particle. In real observations, an average of all particles in the radar resolution volume will reduce such large variability; additionally, $Z_{\mathrm{HH}}$ and $K_{\mathrm{DP}}$ depend on the number concentration.

To summarize, generally when the hailstone size is around $3 \mathrm{~cm}$, the scattering properties change smoothly with incident direction. However, the various irregular shapes of the real hailstones result in larger variability compared to the corresponding spheroids, especially for asymmetric shapes. When the hailstone size approaches the wavelength, resonance effects become larger for both the spheroids and hailstones. As the hailstone size exceeds the wavelength, very strong resonance effects occur for both the hailstone and spheroid, with the hailstone exhibiting larger, more unpredictable fluctuations than the spheroid.

\section{b. Averages over the orientation distribution}

The correlation coefficient $\rho_{\text {hv }}$ is an important radar variable for hail detection (e.g., Balakrishnan and Zrnić 1990a; Kumjian and Ryzhkov 2008; Kumjian et al. 2010a; Picca and Ryzhkov 2012) and represents the diversity of particle scattering properties within a radar sampling volume (Kumjian 2013). For $\rho_{\mathrm{hv}}$ calculations, a 
distribution of particles or particle properties has to be included. For a monodisperse distribution of particles like that used herein, $\rho_{\mathrm{hv}}$ is a measure of the variability of its backscattering properties (in particular $S_{\mathrm{hh}}$ and $S_{\mathrm{vv}}$ ) owing to different orientations: smaller $\rho_{\mathrm{hv}}$ values indicate greater variability. This can be thought of as a sampling volume containing a population of identical particles with a given orientation distribution. As described above (see Figs. 3 and 4), the particles are assumed to have random orientation in the azimuthal direction (i.e., uniform distribution of $\varphi$ ) and a twodimensional Gaussian distribution in elevation angle $\theta$ and canting angle $\alpha$ (e.g., Ryzhkov 2001; Ryzhkov et al. 2011). The width of this orientation distribution is given by the standard deviation $\sigma$. This orientation distribution setup corresponds with a horizontally viewing radar (i.e., $\hat{k}$ is in the horizontal plane) observing particles with a preferred orientation in the horizontal plane.

We emphasize that this is a limitation of our calculations. In real storms, the $\rho_{\mathrm{hv}}$ represents a measure of diversity of all particles in the sampling volume. Because we lack a sufficiently large number of real hailstones in the 3D scanner database to construct a PSD (and because hail PSDs are highly uncertain), our $\rho_{\text {hv }}$ values herein are best thought of as a measure of a given hailstone's scattering property variability. It offers some insights on how much $\rho_{\text {hv }}$ may be reduced by irregularities of such stones but should not be taken as an indication of what the true observed $\rho_{\mathrm{hv}}$ may be in real storms, unless the sampling volume is full of particles with similar irregular shapes. One limitation with real observations is that only a limited number (usually 32 or 64) of pulses are recorded. Therefore, $\rho_{\mathrm{hv}}$ in real measurements is an estimation based on a stochastic process. A simple test was performed to see how $\rho_{\text {hv }}$ fluctuates with different number of pulses and is provided in the appendix.

Figure 7 shows $\rho_{\mathrm{hv}}$ as a function of $\sigma$. The last marked value is for a random orientation distribution (rand), which gives the lowest $\rho_{\mathrm{hv}}$ value of that particle. When hailstones are relatively small compared to the wavelength ( $\sim 3 \mathrm{~cm}$ in size; hailstones $1-5), \rho_{\mathrm{hv}}$ is close to 1 . More irregular shapes cause additional variability such that $\rho_{\mathrm{hv}}$ can drop close to 0.96 (e.g., hailstone 4). However, interestingly, the "spiky" hailstone 5 produces high $\rho_{\text {hv }}(>0.98)$ for all $\sigma$. In comparison, the smooth spheroidal approximations for these small hailstones produce larger $\rho_{\mathrm{hv}}$ for a given $\sigma$. Thus, despite being small compared to the wavelength, the irregularities of hailstone shapes can reduce $\rho_{\mathrm{hv}}$ compared to their corresponding spheroid approximations.
For hailstones 6 and 7, their size is about the same as the wavelength. Although hailstones 6 and 7 are giant, the $\rho_{\mathrm{hv}}$ values are not as dramatically reduced as one may expect: minimum values for hailstones 6 and 7 are 0.94 and 0.97 , respectively. Recall that so far the hailstones are considered dry for our scattering calculations: adding liquid to the surface will further decrease the $\rho_{\mathrm{hv}}$ owing to the increase in apparent diversity of scattering properties when increasing the relative permittivity (Kumjian 2013; also see below). Hailstone 8 has a size of about $1.5 \lambda$. Its scattering properties exhibit huge variability over incident radiation directions compared with the corresponding spheroid. Thus the correlation coefficient can get as low as 0.56 for random orientation, whereas the spheroid only reaches 0.86 for random orientation. Both of these minimum values are lower than any other particle in the dataset, and are comparable to values characterizing nonmeteorological scatterers.

These calculations demonstrate that, even for large particles for which resonance scattering occurs, spheroids often fail to reproduce the observed low $\rho_{\mathrm{hv}}$ values by just considering the scattering variability due to orientation (e.g., Picca and Ryzhkov 2012). For dry hailstones, such substantially reduced $\rho_{\mathrm{hv}}(<0.9)$ can occur in the presence of large, irregularly shaped particles. In contrast, the small, spiky hailstone (hailstone 5) does not produce a large reduction in $\rho_{\mathrm{hv}}$. However, highly irregularly shaped small hailstones (like hailstone 4) can produce similar $\rho_{\text {hv }}$ reductions to large, resonance-sized particles (cf. hailstone 4 and hailstones 6 and 7). As will be discussed later, the addition of liquid water on the hailstones will further decrease $\rho_{\mathrm{hv}}$, regardless of size. Together, these calculations suggest that hail size information from $\rho_{\mathrm{hv}}$ reductions could be ambiguous. In real measurements, not only the fluctuation of scattering properties with respect to orientation, but also the presence of particles with different shapes in the sampling volume, will decrease $\rho_{\mathrm{hv}}$. To the extent that real hail PSDs comprise irregularly shaped and/or nonspheroidal stones, $\rho_{\text {hv }}$ can be substantially reduced even without the presence of significantly severe hail.

Kennedy et al. (2001) found enhanced LDR values $(>-25 \mathrm{~dB})$ aloft in storms that produced damaging hail at the surface. They suggested that enhanced LDR values within the hail growth region aloft may be a good indicator of large hail. Using scattering calculations for smooth spheroids, they suggested that the presence of liquid water on growing hailstones was the cause of the enhanced LDR values. To explore the LDR signatures associated with realistic hailstones, we compute LDR for each of the dry hailstones in the dataset. 

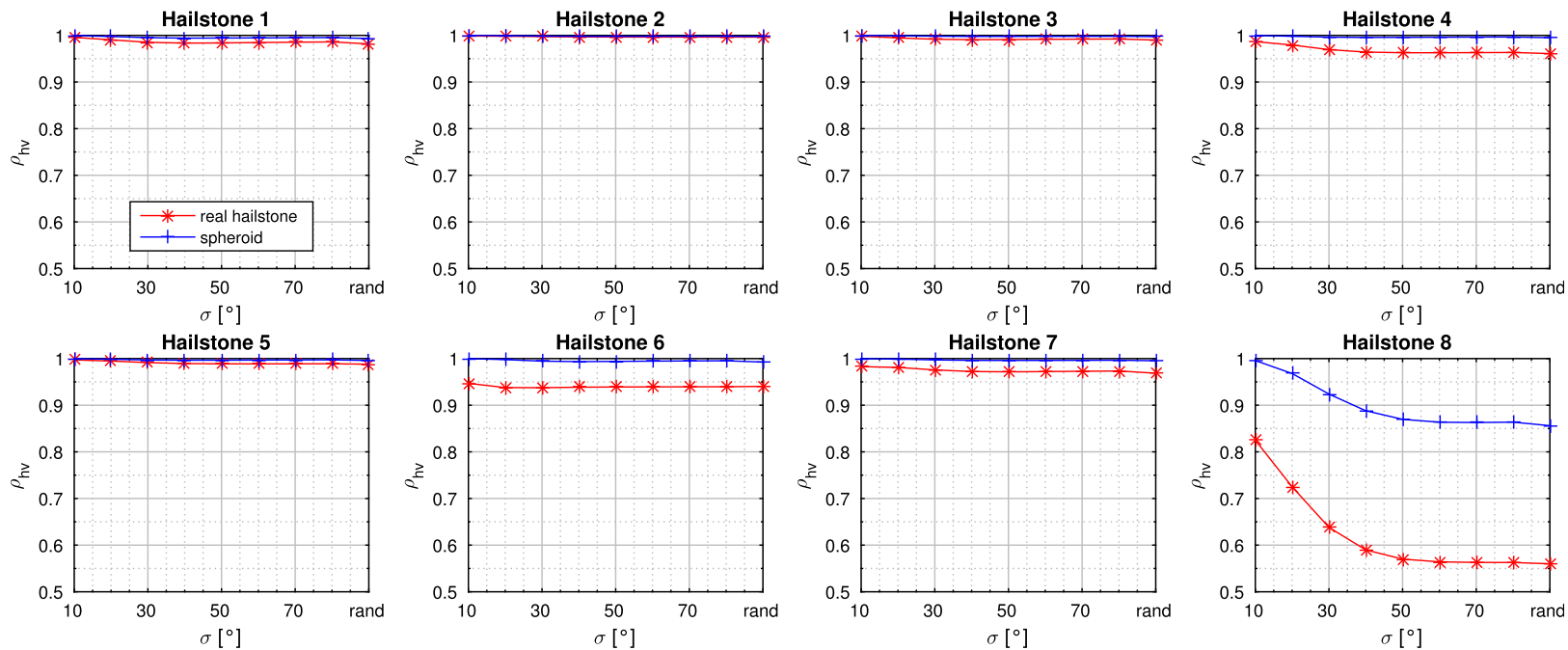

FIG. 7. Correlation coefficient $\rho_{\mathrm{hv}}$ as a function of the standard deviation of canting angles for all eight hailstones (red) compared to that from the corresponding spheroids (blue).

Figure 8 shows the LDR for each hailstone as a function of $\sigma$. It is found that LDR changes less than $5 \mathrm{~dB}$ because of orientations even for large irregular hailstones, but it does have a dependence on size. Therefore, LDR could be a potential size indicator of hailstones, as suggested by Kennedy et al. (2001). However, the dependence of LDR on size is rather ambiguous in that LDR cannot be used solely to indicate size. Meanwhile, the smooth spheroids have lower LDR for most $\sigma$, in most cases $5-10 \mathrm{~dB}$ below the values for the real hailstones. This suggests that any hail-sizing algorithms developed using LDR or a proxy measurement from simultaneous transmission and reception polarimetric radars (Ryzhkov et al. 2017) should account for these higher values compared to what is provided by calculations using smooth spheroids.

Because the variation of scattering properties can be extreme for large and giant hailstones, a natural question arises: how large might the variability be in polarimetric radar measurements with a given number of particles in the radar resolution volume? We perform a third set of calculations to investigate how the number of hailstones in the radar volume influences the variability of each polarimetric radar observable. This can be thought of as trying to characterize the uncertainty associated with not fully sampling all orientation angles in a given sampling volume, which was done for the second set of calculations. To do so, a reference
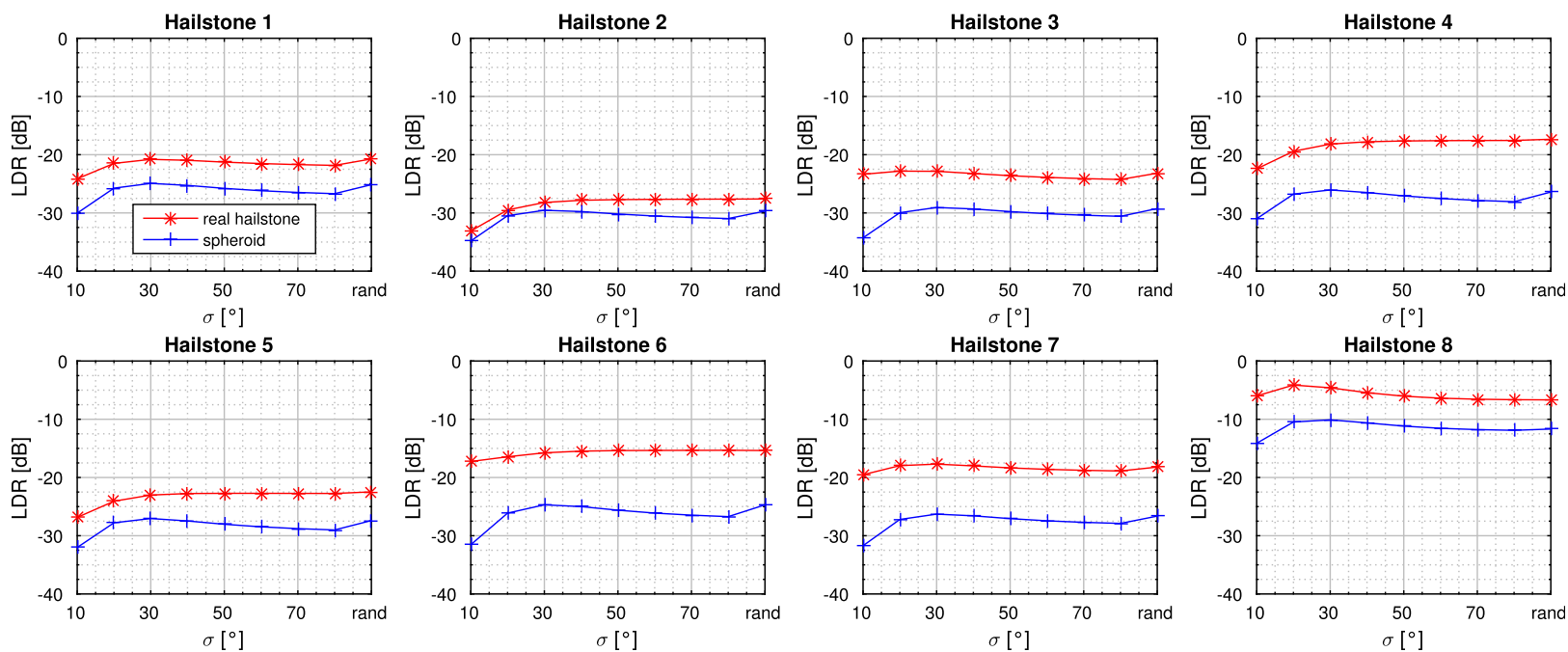

FIG. 8. As in Fig. 7, but for LDR. 
volume and a reference number of hailstones are set as follows. The resolution length along the propagation direction is about $250 \mathrm{~m}$ and the beamwidth is $0.9^{\circ}$ for S-band Weather Surveillance Radar-1988 Doppler (WSR-88D). The reference resolution volume is set as $4 \times 10^{7} \mathrm{~m}^{3}$, corresponding to $25.5-\mathrm{km}$ distance from the radar. The reference number of hailstones is set at 100 . However, $Z_{\mathrm{HH}}$ and $K_{\mathrm{DP}}$ are affected by the number concentration of particles. Therefore, for each given number of hailstones in the radar resolution volume, $Z_{\mathrm{HH}}$ and $K_{\mathrm{DP}}$ are normalized to the reference number of hailstones, so that an easy comparison can be made between different sampling numbers. This reference number is set arbitrarily with the normalized $Z_{\mathrm{HH}}$ and $K_{\mathrm{DP}}$ in a reasonable range. It is worth noting that the radar resolution volume increases with increasing distance to radar. For a given number concentration, the number of hailstones in the radar volume is larger farther away from the radar.

Different numbers of hailstones in the radar resolution volume are tested. For each given number of particles $N^{*}$ in the radar resolution volume, $N^{*}$ orientation sets are sampled (incident radiation elevation angles $\theta$ and canting angles $\alpha$ are sampled following a Gaussian distribution with $0^{\circ}$ mean and $40^{\circ}$ standard deviation, and azimuth angles $\varphi$ are sampled uniformly). Then each of the polarimetric radar variables can be calculated following Eqs. (7)-(12). These steps are repeated 1000 times to see how large the variation in each polarimetric radar variable is. The idea is that the full variability of natural hailstone scattering properties likely will not be sampled owing to their relatively low concentrations; these calculations aim to quantify the expected uncertainty in observations of irregular hailstones. Such uncertainties in scattering properties can inform traditional forward operators and models with spheroidal particles to try and better capture natural variability. Note that we perform these sampling statistics for spheroids as well to characterize the "lower bound" of uncertainties for these particles.

The results for several selected hailstones are shown in Figs. 9 and 10, which display 2D histograms with the abscissa as the number of particles in the radar volume and the ordinate as the value of the polarimetric radar variable. In general, the spread of values of the polarimetric radar variables is larger for hailstones than for the spheroids. The $Z_{\mathrm{HH}}$ variation with orientation averaging of a limited number of samples is smaller than $5 \mathrm{~dB}$ for the spheroids, but can be $>10 \mathrm{~dB}$ for hailstone 8 . For the spheroids, the $Z_{\mathrm{DR}}$ variation is about $2 \mathrm{~dB}$ when the size is about $3 \mathrm{~cm}$ (Fig. 9), less than $1.5 \mathrm{~dB}$ when the size is about $10 \mathrm{~cm}$ (spheroids 6 and 7 in Fig. 10) owing to the larger aspect ratio, but $>5 \mathrm{~dB}$ for spheroid 8 because of strong resonance scattering effects. However, the real hailstones can have 1.5-2 times more variation in $Z_{\mathrm{DR}}$ than the spheroids. The $K_{\mathrm{DP}}$ variability behaves similarly to that of $Z_{\mathrm{DR}}$. We notice that the mean $Z_{\mathrm{DR}}$ and $K_{\mathrm{DP}}$ values tend to be positive for small particles but slightly negative for large particles. The variability of LDR is similar for both the spheroids and hailstones, but the hailstones have consistently higher LDR, as mentioned above. The value of $\rho_{\text {hv }}$ is very close to 1 for spheroids with sizes $\sim 3 \mathrm{~cm}$, with very little variation. In contrast, the variability of $\rho_{\text {hv }}$ for the $\sim 3-\mathrm{cm}$ hailstones is larger. For larger particles (Fig. 10, hailstones 6 and 7), $\rho_{\mathrm{hv}}$ is also very close to 1 , and the real hailstones have larger variability than their spheroidal counterparts. However, when the particle size is $>15 \mathrm{~cm}$ (Fig. 10, hailstone 8), $\rho_{\text {hv }}$ drops to 0.6 and exhibits huge variability, whereas spheroids still have $\rho_{\mathrm{hv}}$ near 0.9 with about 0.2 variation. Owing to the limited number of pulses recorded operationally by radars, the random phase in backscattering owing to particle locations radially within the sampling volume may not average to zero as is typically assumed (see the appendix). However, in this paper, we focus on the variability of the scattering properties themselves. In this case, $\rho_{\mathrm{hv}}$ calculated here represents the value by only considering the scattering variability of a single hailstone owing to different orientations. Even so, we see that in certain situations, $\rho_{\text {hv }}$ can be well below 0.85 [the lower bound for hail classification in the hydrometeor classification algorithm of Park et al. (2009)] without considering the random-phase part of the backscatter. This result is useful for hail detection, because hail can have rather low $\rho_{\text {hv }}$, but some hydrometeor classification algorithms might classify it as nonmeteorological echoes.

\section{c. Liquid-coated hailstones}

So far, we have only considered dry hailstones. However, as hailstones descend below the altitude of the $0^{\circ} \mathrm{C}$ wet-bulb temperature, they begin to melt and can retain a film of liquid water on their surface (e.g., Rasmussen et al. 1984; Rasmussen and Heymsfield 1987). The same applies to hailstones undergoing wet growth in the updrafts of severe convective storms. Although typically treated as a thin liquid coating of uniform thickness in scattering calculations (e.g., Snyder et al. 2010; Ryzhkov et al. 2011, 2013a), this coating of liquid may be distributed unevenly on the surface of natural hailstones and may change depending on fall behavior and orientation (Rasmussen et al. 1984). Additionally, note that small $(<2 \mathrm{~cm})$ melting hailstones are thought to retain substantial amounts of liquid water that can acquire a "torus" shape with an aspect ratio that 

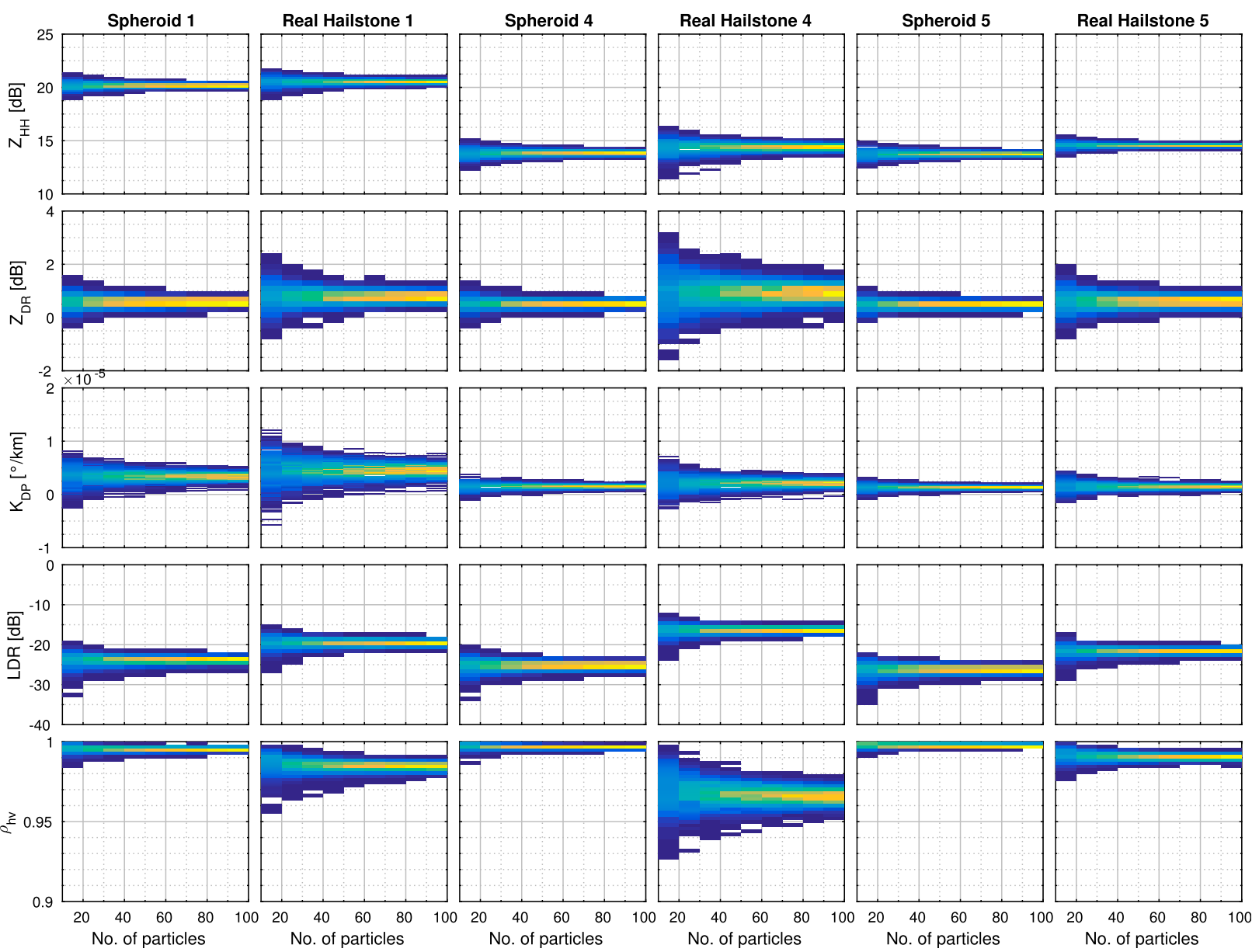

low

FIG. 9. 2D histograms of each polarimetric variable $\left(Z_{\mathrm{HH}}, Z_{\mathrm{DR}}, K_{\mathrm{DP}}, \mathrm{LDR}\right.$, and $\left.\rho_{\mathrm{hv}}\right)$ and the number of particles in the radar volume for hailstones 1,4 , and 5 , with yellow and blue representing high and low probability, respectively.

is different than the embedded ice core (Rasmussen et al. 1984). Given these uncertainties and for simplicity, here we consider a liquid layer with 1 -mm thickness added to the real hailstones. To improve scattering calculation accuracy, the dipole size is changed to $0.25 \mathrm{~mm}$. The computation time increases dramatically for these large particles with such high resolution. Owing to the limitation of our supercomputing resources, the scattering calculations for liquid-coated hailstones are only performed for the first five hailstones. A two-layer T-matrix code (Bringi and Seliga 1977a,b) is used for liquid-coated spheroids.

Similar to Fig. 5, Fig. 11 shows $Z_{\mathrm{HH}}, Z_{\mathrm{DR}}, K_{\mathrm{DP}}$, and $\delta$ as a function of elevation angle for the liquid-coated hailstones 1-5. Comparing Figs. 5 and 11, it is clear that all polarimetric radar variables are enhanced by adding the liquid layer. The value of $Z_{\mathrm{HH}}$ is significantly increased by $5-10 \mathrm{~dB}$ (especially for hailstones 1,4 , and 5), which is expected given the change in relative permittivity. The variability of each radar variable becomes larger as well. Interestingly, the pattern of $Z_{\mathrm{HH}}$ and $K_{\mathrm{DP}}$ as a function of elevation angle for real hailstones 1-3 changes compared to the corresponding dry hailstones. The value of $Z_{\mathrm{HH}}$ is larger at horizontal incidence (elevation angle $\sim 0^{\circ}$ ) than at vertical incidence (elevation angles $-90^{\circ}$ and $90^{\circ}$ ) for hailstones 1-3 with liquid layer, whereas the opposite is true for the dry hailstones. The plot of $K_{\mathrm{DP}}$ has two well-separated peaks for wet hailstones $1-3$, owing to their asymmetry. In contrast, the dry stones have only one $K_{\mathrm{DP}}$ peak at around $0^{\circ}$ elevation angle (i.e., horizontal incidence). The $Z_{\mathrm{DR}}$ values for the liquid-coated hailstones are significantly increased over the values for the dry hailstones owing to the increase in relative permittivity. Similarly, the range and variability in $\delta$ is significantly larger for the liquid-coated hailstones. These calculations imply that some of the extreme values of the polarimetric variables observed in severe convective 


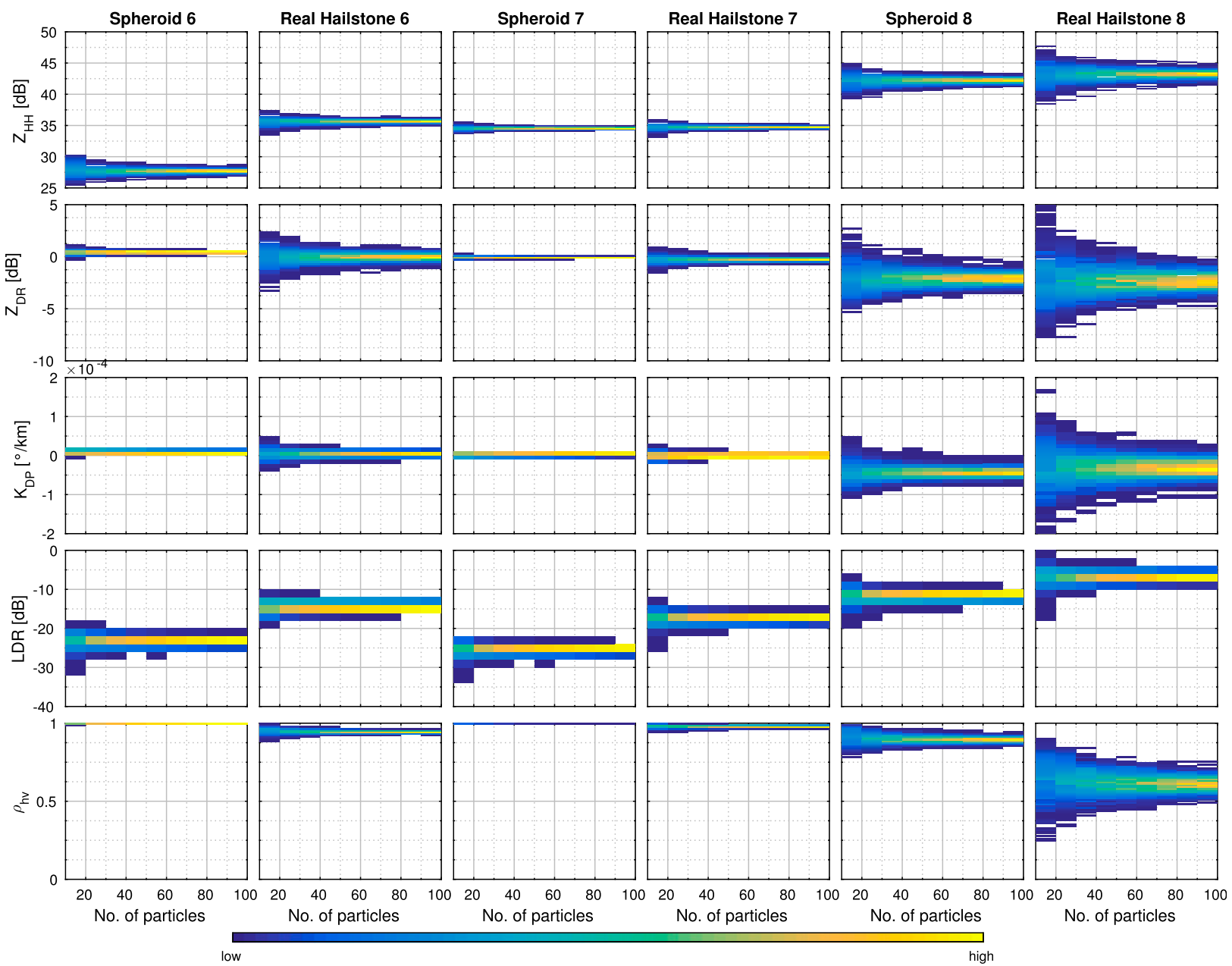

FIG. 10. As in Fig. 9, but for hailstones 6-8.

storms (e.g., $Z_{\mathrm{DR}}>5 \mathrm{~dB}$, regions of $\delta$ and/or significantly reduced $\rho_{\text {hv }}$ ) may arise from irregularly shaped hailstones with thin liquid coatings. For the liquidcoated spheroids, the patterns and variability in the polarimetric variables generally is unable to match those computed for the real hailstones. Note that particle 4 exhibits strong resonance effects, which is unexpected. One reason is that the aspect ratio of hailstone 4 is about the limit of what can be handled in the two-layer T-matrix code. So, the two-layer T-matrix code results for particle 4 are hereafter excluded from discussion.

Figure 12 shows $\rho_{\mathrm{hv}}$ and LDR as a function of canting standard deviation $\sigma$ for wet hailstones. As expected, owing to the increase of variability of scattering properties by adding the liquid layer, $\rho_{\text {hv }}$ drops substantially compared to the dry hailstones. Adding a liquid layer increases the LDR by about $10 \mathrm{~dB}$ for both real hailstones and spheroids. Therefore, the coated liquid layer on the hailstone needs to be considered when using LDR or its proxy to indicate size.

\section{Conclusions and discussion}

Three-dimensional scans of real hailstones by Giammanco et al. (2017) are used to calculate S-band hailstone scattering properties using the DDA method. The results are compared with the hailstones modeled as spheroids with same maximum and minimum dimensions and mass. Such spheroids are widely used for scattering calculations of nonspherical hydrometeors in the field. The main focus of this study is to investigate how the various irregular shapes of real hailstones affect their scattering properties compared with symmetric spheroids.

As expected, owing to their various shapes and irregularities, real hailstones have larger variability of $Z_{\mathrm{HH}}$ than spheroids. The number of particles in the 

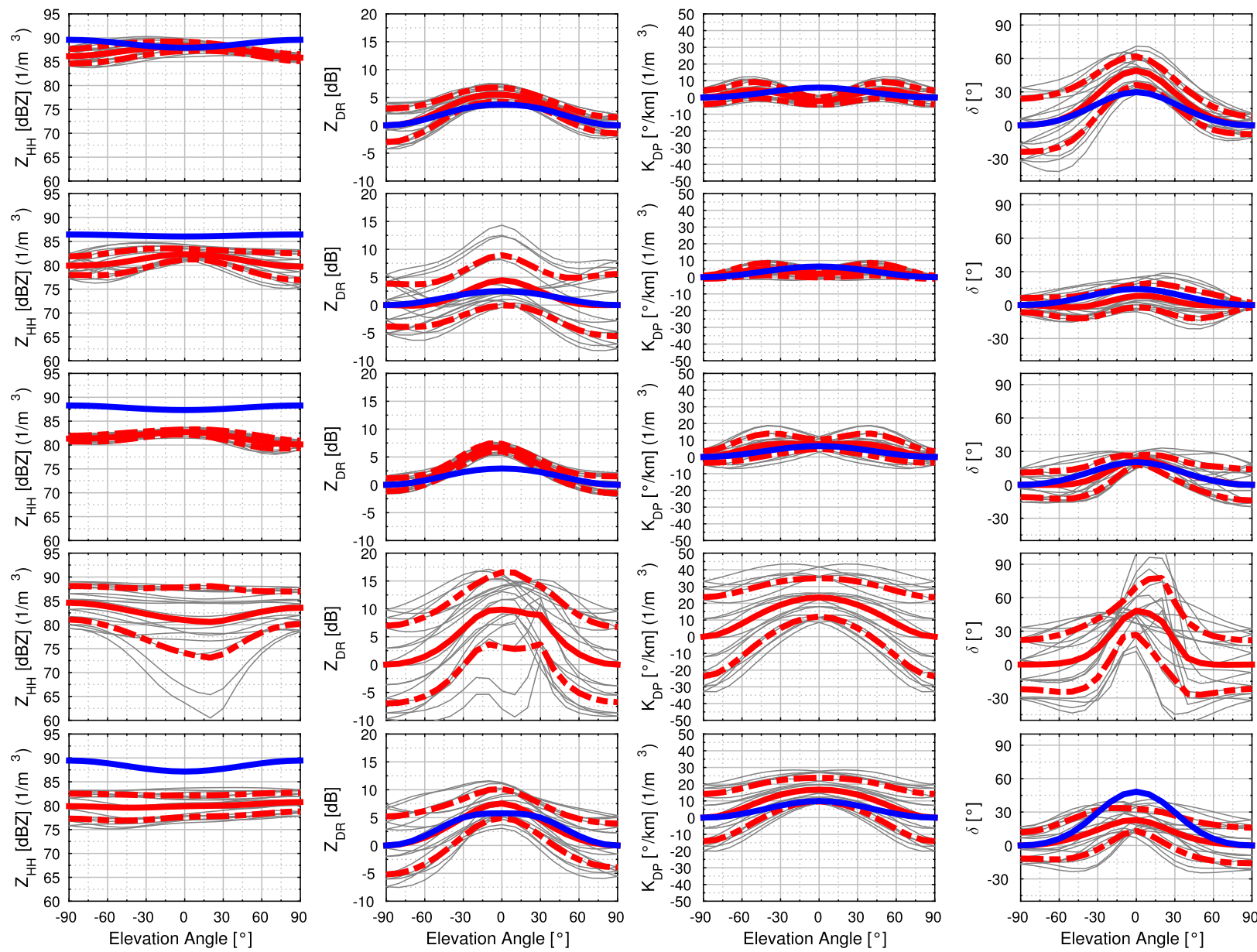

FIG. 11. As in Fig. 5, but with a 1-mm liquid coating.

radar resolution volume determines this variability. On average, the difference of $Z_{\mathrm{DR}}$ between dry real hailstones and spheroids is small. In one case (hailstone 8 in Fig. 10), the spheroid has $Z_{\mathrm{DR}}$ around $-2 \mathrm{~dB}$, whereas the real hailstone $Z_{\mathrm{DR}}$ is around $-3 \mathrm{~dB}$. Such values are consistent with observations of negative $Z_{\mathrm{DR}}$ aloft in the cores of convective updrafts, where such large hailstones form (e.g., Kumjian and Ryzhkov 2008; Kumjian et al. 2010b, 2014). Knight and Knight (1970a) stated that hailstones tend to fall with their minor axis aligned in the horizontal on average, thus giving negative $Z_{\mathrm{DR}}$ for smaller hailstones in which resonance effects are not significant. However, giant hailstones could have negative $Z_{\mathrm{DR}}$ if their minor axis is aligned in the vertical, as shown in this study, which agrees with the calculations of Aydin and Zhao (1990) and Depue et al. (2007). When a 1-mm liquid coating is added, some of the real hailstones exhibit much larger $Z_{\mathrm{DR}}$ values than the corresponding spheroids. Such irregularly shaped, wet particles may help explain the extremely large $(>5 \mathrm{~dB}) Z_{\mathrm{DR}}$ values sometimes observed in severe convective storms (e.g.,
Kumjian and Ryzhkov 2008) that cannot be reproduced in scattering calculations or forward operators employing liquid-coated spheroids (Jung et al. 2008; Ryzhkov et al. 2011).

The magnitude of $K_{\mathrm{DP}}$ is larger for real hailstones than for spheroids. Further, the shape of real hailstones increases the variability of $K_{\mathrm{DP}}$. Therefore, the orientation-averaged $K_{\mathrm{DP}}$ varies more for real hailstones than for spheroids. Owing to the large variability in $\delta$ of real hailstones, it is likely that the presence of large, irregularly shaped hailstones with significant $\delta$ will make the differential phase shift measurements noisy and $K_{\mathrm{DP}}$ estimation difficult if not impossible. Further, in operational radar data, $K_{\mathrm{DP}}$ is censored where $\rho_{\text {hv }}$ is significantly reduced. Large variability of $\delta$ in the sampling volume will reduce $\rho_{\mathrm{hv}}$, possibly leading to $K_{\mathrm{DP}}$ data censoring. Nonuniform beamfilling, which can be significant in supercells and other hailstorms, also causes errors in $K_{\text {DP }}$ estimation (e.g., Ryzhkov 2007; Kumjian and Ryzhkov 2008). Therefore, accurate $K_{\mathrm{DP}}$ estimation in the presence of large 

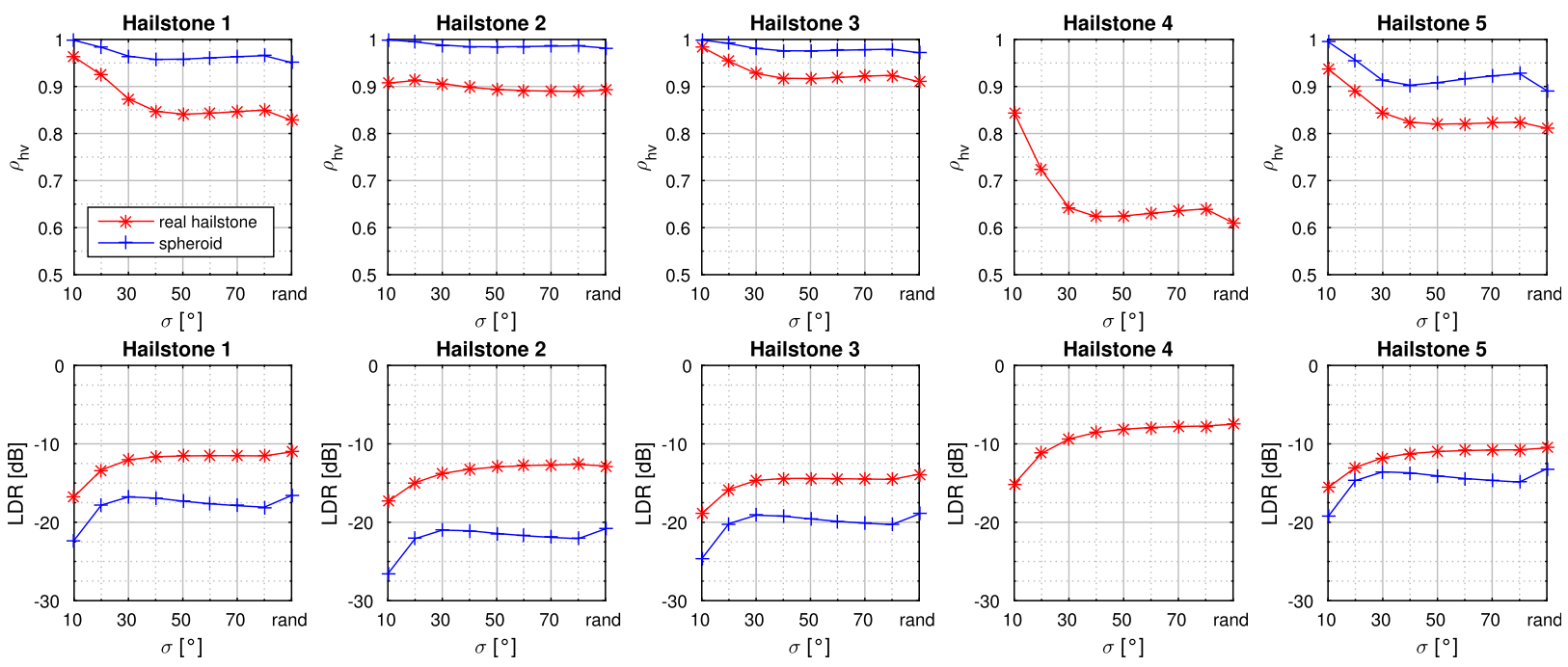

FIG. 12. As in Figs. 7 and 8, but for hailstones 1-5 with a 1-mm liquid coating.

hailstones will be challenging, exhibiting large variability (e.g., Picca and Ryzhkov 2012).

In general, $\rho_{\text {hv }}$ is lower for natural hailstone shapes than for spheroids. The $\rho_{\mathrm{hv}}$ values generally remained above about 0.9 for all dry hailstones and spheroids considered herein. A notable exception is the Vivian, South Dakota, hailstone (hailstone 8), which produces dramatically reduced $\rho_{\text {hv }}$ as low as 0.56 , well within the range observed for tornadic debris and other nonmeteorological scatterers. The wet realistic hailstones produced lower $\rho_{\text {hv }}(0.8-0.92)$ for canting angle distribution widths greater than $30^{\circ}$, except hailstone 4 with $\rho_{\text {hv }}$ well below 0.8 most times. Although the liquidcoated spheroids have lower $\rho_{\mathrm{hv}}$ than their dry counterparts, values are still typically $\sim 0.1$ higher than for the realistic wet hailstones. These differences could explain why many observations reveal quite low $\rho_{\text {hv }}$ (e.g., Kumjian and Ryzhkov 2008; Picca and Ryzhkov 2012; Kaltenboeck and Ryzhkov 2013), whereas radar forward operators employing spheroid models fail to produce such low $\rho_{\mathrm{hv}}$ values (Jung et al. 2010; Ryzhkov et al. 2011; Kumjian et al. 2012, 2014; Johnson et al. 2016).

Both realistic hailstones and spheroids exhibit a correlation between size and LDR, whereas real hailstones have $5-10 \mathrm{~dB}$ higher LDR than their spheroidal counterparts. Once a $\sim 1-\mathrm{mm}$ liquid layer is added to the particle, the LDR values are increased by around $10 \mathrm{~dB}$. Although LDR seems to be a good indicator of hail size, whether the hailstone is dry or not needs to be determined in advance. Its location in the storm and/or relative to the $Z_{\mathrm{DR}}$ column or updraft could be useful in this regard.

Mirković (2015) also studied the polarimetric radar variables for artificial nonspheroidal hailstones, and found that negative $Z_{\mathrm{DR}}$ occurs at large sizes and $\rho_{\mathrm{hv}}$ decreases below 0.6 at specific sizes. Even though the same pattern cannot be found from current study owing to the limited number of hailstones, the negative $Z_{\mathrm{DR}}$ and low $\rho_{\mathrm{hv}}$ are indeed captured for the larger ones. In addition to the orientation-averaged polarimetric variables for real hailstones, the current study also presented the variability using the scattering properties from over 300 incident-radiation directions for each hailstone. This is critical because it is likely that observations of hail (particularly large or giant hail) will not exhibit this orientation-averaged value because a given sampling volume has only a limited concentration of hailstones, each possibly with unique shapes. This is the first time the variability in scattering properties and the polarimetric radar variables of realistic hailstones is calculated in this way. Such variability can be taken as a proxy for the uncertainty in scattering calculations used in forward operators and hail size retrievals.

One of the greatest uncertainties affecting the polarimetric radar variables is how the hailstones are oriented while falling (e.g., Heymsfield et al. 2014). The uncertainty is compounded by not knowing the liquid meltwater morphology on such falling irregular hailstones. The simple assumption used in this study of a 1-mm liquid coating at least provides an indication of the effects on scattering of liquid on the surface of irregular hailstones. Testing of hailstone fall behavior using 3D prints of the scanned hailstones is underway and is expected to provide key insights to help constrain the scattering calculations. In the meantime, scattering properties for multiple incident radiation directions should be calculated and saved so that the different orientation distribution assumptions may be tested. 
Owing to the limited number of hailstones available for this study, a direct comparison with real radar observations by integrating over a realistic hail size distribution is impossible. Here, a monodisperse distribution is used to convert the single scattering properties into polarimetric radar variables. Therefore, the values of the polarimetric radar variables are not representative of real radar observations, and the scattering variability of a single particle does not represent the variability within a realistic radar volume. The main focus here is on the difference of scattering properties between the real hailstones and spheroids. As the IBHS annual field project continues, we expect the database of 3D-scanned hailstones to grow as various sizes will become available. Therefore, a scattering database for real hailstones should be built in a similar manner to the Pennsylvania State University database for pristine ice crystals and aggregates (Lu et al. 2016). Such a database for hail will provide valuable information about the mean of each polarimetric variable and the variability, which could benefit radar forward operators and data assimilation efforts, as well as hail detection.

The hailstones considered herein cover a wide range of sizes. The large variability in scattering properties revealed by our calculations, especially for the largest hailstones, has implications for radar-based hail-sizing efforts. For example, for some incident radiation directions, the record Vivian, South Dakota, hailstone produces similar $Z_{\mathrm{HH}}$, as some of the smaller hailstones in the dataset for an identical number concentration. Given that smaller stones are much more likely to be present in higher concentrations, $Z_{\mathrm{HH}}$ could reasonably be higher in the case of small hail than giant hail, not to mention contributions from rain originating from shedding and complete melting of smaller hailstones. The fall behavior of irregular hailstones is highly uncertain, rendering hail size inferences from $Z_{\mathrm{DR}}$ ambiguous. Consistent observations of negative $Z_{\mathrm{DR}}$ in convective storm updraft mixed-phase regions (e.g., Kumjian et al. 2014) are intriguing and should be further explored for their insights into fall behavior of hailstones. LDR exhibits the strongest dependence on hail size but also has a dependence on whether the hailstone is wet or not. In some cases, the combination of $\rho_{\mathrm{hv}}$ and LDR could serve as an indicator of large hail. For instance, based on these calculations, extremely low $\rho_{\mathrm{hv}}$
$(<0.6)$ and high LDR $(>-5 \mathrm{~dB})$ could indicate huge, wet hailstones $(>15 \mathrm{~cm})$. Determination of whether or not a hailstone is wet is needed prior to implementation of any sizing algorithm, similar to Ryzhkov et al. (2013b). Any quantitative hail-sizing algorithms should account for the wider variability of scattering properties of real hailstones as shown herein, instead of relying solely on spheroids.

Acknowledgments. The PSU authors are funded by the National Science Foundation (NSF) through Grants AGS-128180 and AGS-1661679, and a grant from the Insurance Institute for Business and Home Safety (IBHS). The scattering calculations of hailstones were conducted with Advanced Cyberinfrastructure computational resources provided by the Institute for CyberScience at The Pennsylvania State University (http://ics.psu.edu). We thank the three anonymous reviewers for their constructive comments and suggestions that significantly improved the organization and clarity of the manuscript. The National Center for Atmospheric Research is sponsored by the National Science Foundation.

\section{APPENDIX}

\section{Ergodicity as Applied to $\rho_{\mathrm{hv}}$ Calculations}

The correlation coefficient $\rho_{\mathrm{hv}}$ is one of the polarimetric radar variables that is difficult to understand conceptually because it is not readily apparent how the theoretical formula [Eq. (10)] relates to real radar measurements. We relate the theoretically calculated $\rho_{\text {hv }}$ to real observations here. We start with the scattering covariance matrix used in Bringi and Chandrasekar (2001), based on which radar variables are calculated. Then we show the derivations of Eq. (10) using the relations between the scattering covariance matrix and the backscattering amplitude matrix $\mathbf{S}_{\mathrm{BSA}}$ of theoretical calculations, in which the ergodic hypothesis is a key step. Last, we simulate radar measurements by sampling pulses and show the convergence of simulated $\rho_{\mathrm{hv}}$ to the expected value calculated with Eq. (10) as the number of pulses increases.

First, all the radar variables are defined in terms of the elements of the time-averaged scattering covariance matrix [Bringi and Chandrasekar 2001, Eq. (3.176b)]:

$$
\boldsymbol{\Sigma}_{\mathrm{BSA}}=\left(\begin{array}{ccc}
\left\langle\gamma_{\mathrm{hh}}^{2}\right\rangle & \sqrt{2}\left\langle\gamma_{\mathrm{hh}} \gamma_{\mathrm{hv}} e^{j\left(\alpha_{\mathrm{hh}}-\alpha_{\mathrm{hv}}\right)}\right\rangle & \left\langle\gamma_{\mathrm{hh}} \gamma_{\mathrm{vv}} e^{j\left(\alpha_{\mathrm{hh}}-\alpha_{\mathrm{vv}}\right)}\right\rangle \\
\sqrt{2}\left\langle\gamma_{\mathrm{hv}} \gamma_{\mathrm{hh}} e^{j\left(\alpha_{\mathrm{hv}}-\alpha_{\mathrm{hh}}\right)}\right\rangle & 2\left\langle\gamma_{\mathrm{hv}}^{2}\right\rangle & \sqrt{2}\left\langle\gamma_{\mathrm{hv}} \gamma_{\mathrm{vv}} e^{j\left(\alpha_{\mathrm{hv}}-\alpha_{\mathrm{vv}}\right)}\right\rangle \\
\left\langle\gamma_{\mathrm{vv}} \gamma_{\mathrm{hh}} e^{j\left(\alpha_{\mathrm{vv}}-\alpha_{\mathrm{hh}}\right)}\right\rangle & \sqrt{2}\left\langle\gamma_{\mathrm{vv}} \gamma_{\mathrm{hv}} e^{j\left(\alpha_{\mathrm{vv}}-\alpha_{\mathrm{hv}}\right)}\right\rangle & \left\langle\gamma_{\mathrm{vv}}^{2}\right\rangle
\end{array}\right)
$$



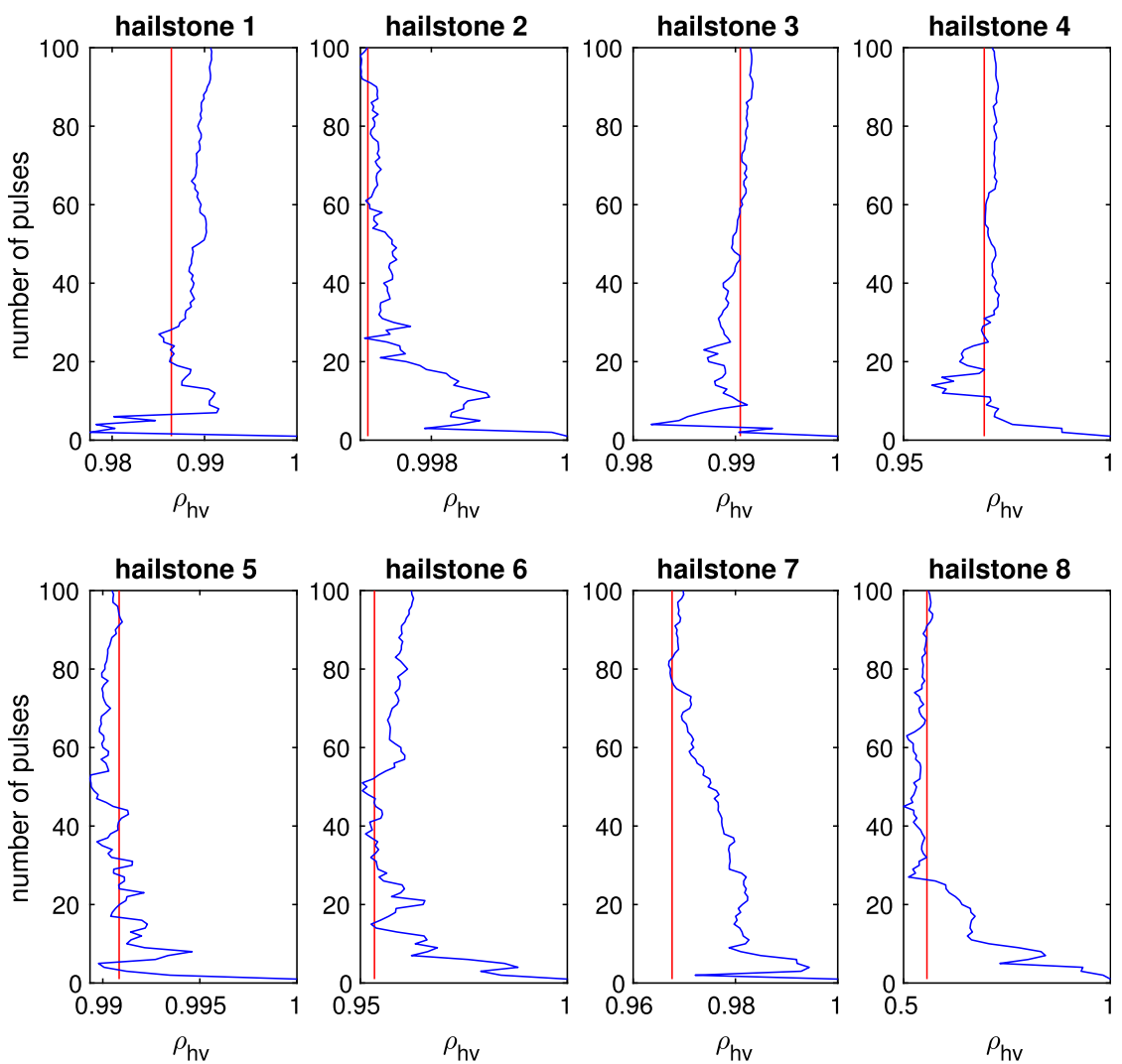

FIG. A1. A simulation of radar signal returns from evenly distributed hailstones within 40 wavelengths of range. The positions of the particles change according to a Gaussian distribution with a spectrum width of $5 \mathrm{~m} \mathrm{~s}^{-1}$, and a pulse repetition time of $5 \mathrm{~ms}$. The corresponding estimates of $\rho_{\mathrm{hv}}$ (blue line) after each additional pulse are shown. As the number of samples increases, the estimates converge toward their expected values (red lines).

where the angle brackets denote the time averaging. The correlation coefficient is calculated as [Bringi and Chandrasekar 2001, Eq. (3.182c)]:

$$
\rho_{\mathrm{hv}}=\frac{\left\langle\gamma_{\mathrm{vv}} \gamma_{\mathrm{hh}} e^{j\left(\alpha_{\mathrm{vv}}-\alpha_{\mathrm{hh}}\right)}\right\rangle}{\sqrt{\left\langle\gamma_{\mathrm{hh}}^{2}\right\rangle\left\langle\gamma_{\mathrm{vv}}^{2}\right\rangle}} .
$$

The backscattering covariance matrix element $\gamma_{\mathrm{hh}, \mathrm{vv}}(t) e^{j \alpha_{\mathrm{hh}, \mathrm{vv}}(t)}$ is related to the backscattering amplitude matrix $S_{\mathrm{hh}, \mathrm{vv}}$ of single particles as follows
[Bringi and Chandrasekar 2001, Eqs. (3.144a) and (3.144b)]:

$$
\gamma_{\mathrm{hh}, \mathrm{vv}}(t) e^{j \alpha_{\mathrm{hh}, \mathrm{vv}}(t)}=\sum_{m} S_{\mathrm{hh}, \mathrm{vv}} e^{j\left(\omega_{m} t+\theta_{m}\right)},
$$

where $m$ loops through all particles in the radar resolution volume. The ergodicity principle is then used to calculate the elements of $\boldsymbol{\Sigma}_{\mathrm{BSA}}$ in terms of $S_{\mathrm{hh}, \mathrm{vv}}$. For brevity, we show the derivation for the element $\left\langle\gamma_{\mathrm{vv}} \gamma_{\mathrm{hh}} e^{j\left(\alpha_{\mathrm{vv}}-\alpha_{\mathrm{hh}}\right)}\right\rangle$ by substituting Eq. (A3).

$$
\begin{aligned}
\left\langle\gamma_{\mathrm{vv}} \gamma_{\mathrm{hh}} e^{j\left(\alpha_{\mathrm{vv}}-\alpha_{\mathrm{hh}}\right)}\right\rangle & =\left\langle\left(\sum_{m} S_{\mathrm{vv}} e^{j\left(\omega_{m} t+\theta_{m}\right)}\right) \times\left(\sum_{m} S_{\mathrm{hh}}^{*} e^{-j\left(\omega_{m} t+\theta_{m}\right)}\right)\right\rangle \\
& =\left\langle\sum_{m} S_{\mathrm{vv}}^{m} S_{\mathrm{hh}}^{m *}+\sum_{l, n}^{l \neq n} S_{\mathrm{vv}}^{l} S_{\mathrm{hh}}^{n^{*}} e^{\left[\left(\omega_{l}-\omega_{n}\right) t+\left(\theta_{l}-\theta_{n}\right)\right]}\right\rangle \\
& =\left\langle\sum_{m} S_{\mathrm{vv}}^{m} S_{\mathrm{hh}}^{m *}\right\rangle+\left\langle\sum_{l, n}^{l \neq n} S_{\mathrm{vv}}^{l} S_{\mathrm{hh}}^{n^{*}} e^{j\left[\left(\omega_{l}-\omega_{n}\right) t+\left(\theta_{l}-\theta_{n}\right)\right]}\right\rangle
\end{aligned}
$$


After pulse averaging, the cross term $\sum_{l, n}^{l \neq n} S_{\mathrm{vv}}^{l} S_{\mathrm{hh}}^{n^{*}} e^{j\left[\left(\omega_{l}-\omega_{n}\right) t+\left(\theta_{l}-\theta_{n}\right)\right]}$ will converge to 0 because the locations of particles within the sampling volume are uncorrelated. So,

$$
\left\langle\gamma_{\mathrm{vv}} \gamma_{\mathrm{hh}} e^{j\left(\alpha_{\mathrm{vv}}-\alpha_{\mathrm{hh}}\right)}\right\rangle=\left\langle\sum_{m} S_{\mathrm{vv}}^{m} S_{\mathrm{hh}}^{m *}\right\rangle
$$

Similarly, for the remaining elements in $\mathbf{\Sigma}_{\mathrm{BSA}}$ [Bringi and Chandrasekar 2001, Eq. (3.183)],

$$
\Sigma_{\mathrm{BSA}}=\left(\begin{array}{ccc}
\left\langle\sum\left|S_{\mathrm{hh}}\right|^{2}\right\rangle & \sqrt{2}\left\langle\sum S_{\mathrm{hh}} S_{\mathrm{hv}}^{*}\right\rangle & \left\langle\sum S_{\mathrm{hh}} S_{\mathrm{vv}}^{*}\right\rangle \\
\sqrt{2}\left\langle\sum S_{\mathrm{hv}} S_{\mathrm{hh}}^{*}\right\rangle & 2\left\langle\sum\left|S_{\mathrm{hv}}\right|^{2}\right\rangle & \sqrt{2}\left\langle\sum S_{\mathrm{hv}} S_{\mathrm{vv}}^{*}\right\rangle \\
\left\langle\sum S_{\mathrm{vv}} S_{\mathrm{hh}}^{*}\right\rangle & \sqrt{2}\left\langle\sum S_{\mathrm{vv}} S_{\mathrm{hv}}^{*}\right\rangle & \left\langle\sum\left|S_{\mathrm{vv}}\right|^{2}\right\rangle
\end{array}\right) .
$$

Therefore, the correlation coefficient $\rho_{\mathrm{hv}}$ becomes

$$
\rho_{\mathrm{hv}}=\frac{\left\langle\sum S_{\mathrm{vv}} S_{\mathrm{hh}}^{*}\right\rangle}{\sqrt{\left\langle\sum\left|S_{\mathrm{hh}}\right|^{2}\right\rangle\left\langle\sum\left|S_{\mathrm{vv}}\right|^{2}\right\rangle}}
$$

equivalent to Eq. (10) in this study.

In real radar measurements, $\rho_{\text {hv }}$ estimate is a stochastic process, because only a limited number of pulses are recorded and the cross term $\sum_{l, n}^{l \neq n} S_{\mathrm{vv}}^{l} S_{\mathrm{hh}}^{n^{*}} e^{j\left[\left(\omega_{l}-\omega_{n}\right) t+\left(\theta_{l}-\theta_{n}\right)\right]}$ may not converge to 0 yet. Here we performed a test similar to Schrom (2018) to illustrate how $\rho_{\text {hv }}$ converges to the expected value as the number of pulses increases. The hailstone is assumed to follow the same orientation distribution as described in section $3 \mathrm{~b}$, with a standard deviation $\sigma$ of $40^{\circ}$. One hundred particles with orientations sampled from the orientation distribution were placed randomly in range within a distance of 40 wavelengths $(100 \mathrm{~mm}$ at $\mathrm{S}$ band). Given a spectrum width of $5 \mathrm{~m} \mathrm{~s}^{-1}$ (standard deviation of radial motion following a Gaussian distribution with 0 mean) and a pulse repetition time of $0.005 \mathrm{~s}$, we allowed each particle to randomly "reshuffle" in range after each of 100 total pulses. After each pulse, we calculated the radar signal returns at both horizontal and vertical polarization $\gamma_{\mathrm{hh}, \mathrm{vv}}$. Finally, we estimated the corresponding $\rho_{\text {hv }}$ for the simulated signals after each additional simulated pulse (shown with the blue curve in Fig. A1). The red curve in Fig. A1 shows the expected value from Eq. (A7).

\section{REFERENCES}

Aydin, K., and Y. Zhao, 1990: A computational study of polarimetric radar observables in hail. IEEE Trans. Geosci. Remote Sens., 28, 412-422, https://doi.org/10.1109/TGRS.1990.572906. , T. A. Seliga, and V. Balaji, 1986: Remote sensing of hail with a dual linear polarization radar. J. Climate Appl. Meteor., 25, 1475-1484, https://doi.org/10.1175/1520-0450(1986)025<1475: RSOHWA $>2.0 . \mathrm{CO} ; 2$.

Balakrishnan, N., and D. S. Zrnić, 1990a: Estimation of rain and hail rates in mixed-phase precipitation. J. Atmos. Sci., 47,
565-583, https://doi.org/10.1175/1520-0469(1990)047<0565: EORAHR $>2.0 . \mathrm{CO} ; 2$

$\longrightarrow$, and — 1990b: Use of polarization to characterize precipitation and discriminate large hail. J. Atmos. Sci., 47, 15251540, https://doi.org/10.1175/1520-0469(1990)047<1525:UOPTCP > 2.0.CO;2.

Blair, S. F., D. R. Deroche, J. M. Boustead, J. W. Leighton, B. L. Barjenbruch, and W. P. Gargan, 2011: A radar-based assessment of the detectability of giant hail. Electron. J. Severe Storms Meteor., 6 (7), http://www.ejssm.org/ojs/index.php/ejssm/ article/viewArticle/87.

Bringi, V. N., and T. A. Seliga, 1977a: Scattering from axisymmetric dielectrics or perfect conductors imbedded in an axisymmetric dielectric. IEEE Trans. Antennas Propag., 25, 575-580, https:// doi.org/10.1109/TAP.1977.1141642.

_ , and _ 1977b: Scattering from non-spherical hydrometeors. Ann. Telecommun., 32, 392-397, https://doi.org/10.1007/ BF03003484.

_ , and V. Chandrasekar, 2001: Polarimetric Doppler Weather Radar. 1st ed. Cambridge University Press, 636 pp.

— T. A. Seliga, and K. Aydin, 1984: Hail detection with a differential reflectivity radar. Science, 225, 1145-1147, https:// doi.org/10.1126/science.225.4667.1145.

_ radar measurements in Colorado convective storms. Part II: Hail detection studies. J. Atmos. Sci., 43, 2564-2577, https:// doi.org/10.1175/1520-0469(1986)043<2564:MRMICC>2.0.CO;2.

Chagnon, S. A., D. Chagnon, and S. D. Hilberg, 2009: Hailstorms across the nation: An atlas about hail and its damages. ISWS Contract Rep. 2009-12, 101 pp., https://www.isws.illinois.edu/ pubdoc/CR/ISWSCR2009-12.pdf.

Depue, T. K., P. C. Kennedy, and S. A. Rutledge, 2007: Performance of the hail differential reflectivity $H_{\mathrm{DR}}$ polarimetric radar hail indicator. J. Appl. Meteor. Climatol., 46, 1290-1301, https://doi.org/10.1175/JAM2529.1.

DeVoe, H., 1964: Optical properties of molecular aggregates. I. Classical model of electronic absorption and refraction. J. Chem. Phys., 41, 393-400, https://doi.org/10.1063/ 1.1725879

Doviak, R. J., and D. S. Zrnić, 1993: Doppler Radar and Weather Observations. 2nd ed. Academic Press, 562 pp.

Draine, B. T., and P. J. Flatau, 1994: Discrete-dipole approximation for scattering calculations. J. Opt. Soc. Amer., 11A, 14911499, https://doi.org/10.1364/JOSAA.11.001491.

Giammanco, I. M., B. R. Maiden, H. Estes, and T. M. BrownGiammanco, 2017: Using 3D laser scanning technology to 
create digital models of hailstones. Bull. Amer. Meteor. Soc., 98, 1341-1347, https://doi.org/10.1175/BAMS-D-15-00314.1.

Heymsfield, A. J., I. M. Giammanco, and R. Wright, 2014: Terminal velocities and kinetic energies of natural hailstones. Geophys. Res. Lett., 41, 8666-8672, https://doi.org/10.1002/ 2014 GL062324.

Höller, H., M. Hagen, P. F. Meischner, V. N. Bringi, and J. C. Hubbert, 1994: Life cycle and precipitation formation in a hybrid-type hailstorm revealed by polarimetric and Doppler radar measurements. J. Atmos. Sci., 51, 2500-2522, https:// doi.org/10.1175/1520-0469(1994)051<2500:LCAPFI>2.0.CO;2.

Hubbert, J. C., V. N. Bringi, L. D. Carey, and S. Bolen, 1998: CSUCHILL polarimetric radar measurements from a severe hail storm in eastern Colorado. J. Appl. Meteor., 37, 749-775, https://doi.org/10.1175/1520-0450(1998)037<0749:CCPRMF> 2.0.CO;2.

Jameson, A. R., and E. A. Mueller, 1985: Estimation of propagationdifferential phase shift from sequential orthogonal linear polarization radar measurements. J. Atmos. Oceanic Technol., 2, 133-137, https://doi.org/10.1175/1520-0426(1985)002<0133: EOPDPS $>2.0 . \mathrm{CO} ; 2$.

Jiang, Z., M. Oue, J. Verlinde, E. E. Clothiaux, K. Aydin, G. Botta, and Y. Lu, 2017: What can we conclude about the real aspect ratios of ice particle aggregates from two-dimensional images? J. Appl. Meteor. Climatol., 56, 725-734, https://doi.org/10.1175/ JAMC-D-16-0248.1.

Johnson, M., Y. Jung, D. T. Dawson, and M. Xue, 2016: Comparison of simulated polarimetric signatures in idealized supercell storms using two-moment bulk microphysics schemes in WRF. Mon. Wea. Rev., 144, 971-996, https://doi.org/10.1175/ MWR-D-15-0233.1.

Jung, Y., G. Zhang, and M. Xue, 2008: Assimilation of simulated polarimetric radar data for a convective storm using the ensemble Kalman filter. Part I: Observation operators for reflectivity and polarimetric variables. Mon. Wea. Rev., 136, 2228-2245, https://doi.org/10.1175/2007MWR2083.1.

_- M. Xue, and G. Zhang, 2010: Simulations of polarimetric radar signatures of a supercell storm using a two-moment bulk microphysics scheme. J. Appl. Meteor. Climatol., 49, 146-163, https://doi.org/10.1175/2009JAMC2178.1.

Kaltenboeck, R., and A. V. Ryzhkov, 2013: Comparison of polarimetric signatures of hail at $\mathrm{S}$ and $\mathrm{C}$ bands for different hail sizes. Atmos. Res., 123, 323-336, https://doi.org/10.1016/ j.atmosres.2012.05.013.

Kennedy, P. C., S. A. Rutledge, W. A. Petersen, and V. N. Bringi, 2001: Polarimetric radar observations of hail formation. J. Appl. Meteor., 40, 1347-1366, https://doi.org/10.1175/1520-0450(2001) $040<1347$ :PROOHF $>2.0 . \mathrm{CO} ; 2$.

Knight, C. A., and N. C. Knight, 1970a: The falling behavior of hailstones. J. Atmos. Sci., 27, 672-681, https://doi.org/10.1175/ 1520-0469(1970)027<0672:TFBOH >2.0.CO;2.

_ and - 1970b: Lobe structures of hailstones. J. Atmos. Sci., 27, 667-671, https://doi.org/10.1175/1520-0469(1970)027<0667: $\mathrm{LSOH}>2.0 . \mathrm{CO} ; 2$.

Kumjian, M. R., 2013: Principles and applications of dualpolarization weather radar. Part I: Description of the polarimetric radar variables. J. Operational Meteor., 1, 226-242, https://doi.org/10.15191/nwajom.2013.0119.

, and Z. J. Lebo, 2016: Large accumulations of small hail. 28th Conf. on Severe Local Storms, Portland, OR, Amer. Meteor. Soc., 8A.2, https://ams.confex.com/ams/28SLS/webprogram/ Paper301237.html.
— , and A. V. Ryzhkov, 2008: Polarimetric signatures in supercell thunderstorms. J. Appl. Meteor. Climatol., 47, 1940-1961, https:// doi.org/10.1175/2007JAMC1874.1.

—, J. C. Picca, S. Ganson, A. V. Ryzhkov, J. Krause, D. Zrnić, and A. Khain, 2010a: Polarimetric radar characteristics of large hail. 25th Conf. on Severe Local Storms, Denver, CO, Amer. Meteor. Soc., 11.2, https://ams.confex.com/ams/ pdfpapers/176043.pdf.

, A. V. Ryzhkov, V. M. Melnikov, and T. J. Schuur, 2010b: Rapid-scan super-resolution observations of a cyclic supercell with a dual-polarization WSR-88D. Mon. Wea. Rev., 138, 3762-3786, https://doi.org/10.1175/2010MWR3322.1.

_ S. M. Ganson, and A. V. Ryzhkov, 2012: Raindrop freezing in deep convective updrafts: A microphysical and polarimetric model. J. Atmos. Sci., 69, 3471-3490, https://doi.org/10.1175/ JAS-D-12-067.1.

- A. P. Khain, N. BenMoshe, E. Ilotoviz, A. V. Ryzhkov, and V. T. J. Phillips, 2014: The anatomy and physics of $Z_{\mathrm{DR}}$ columns: Investigating a polarimetric radar signature with a spectral bin microphysical model. J. Appl. Meteor. Climatol., 53, 1820-1843, https://doi.org/10.1175/JAMC-D-13-0354.1.

- Z. J. Lebo, and A. Ward, 2019: Storms producing large accumulations of small hail. J. Appl. Meteor. Climatol., https:// doi.org/10.1175/JAMC-D-18-0073.1, in press.

Kunkel, K. E., and Coauthors, 2013: Monitoring and understanding trends in extreme storms: State of knowledge. Bull. Amer. Meteor. Soc., 94, 499-514, https://doi.org/10.1175/BAMS-D11-00262.1.

Leitao, M. J., and P. A. Watson, 1984: Application of dual linearly polarized radar data to prediction of microwave path attenuation at 10-30 GHz. Radio Sci., 19, 209-221, https://doi.org/ 10.1029/RS019i001p00209.

Lu, Y., Z. Jiang, K. Aydin, J. Verlinde, E. E. Clothiaux, and G. Botta, 2016: A polarimetric scattering database for non-spherical ice particles at microwave wavelengths. Atmos. Meas. Tech., 9, 5119-5134, https://doi.org/10.5194/ amt-9-5119-2016.

Mirković, D., 2015: Computational electromagnetic applied to scattering observed by polarimetric weather radar. Ph.D. dissertation, School of Meteorology, University of Oklahoma, 241 pp.

Mishchenko, M. I., 2000: Calculation of the amplitude matrix for a nonspherical particle in a fixed orientation. Appl. Opt., 39, 1026-1031, https://doi.org/10.1364/AO.39.001026.

National Weather Service-San Antonio, 2016: San Antonio hail April 2016 weather event summary. Austin/San Antonio Weather Forecast Office, 5 pp., https://www.weather.gov/ media/ewx/wxevents/ewx-20160412.pdf.

Ortega, K. L., J. M. Krause, and A. V. Ryzhkov, 2016: Polarimetric radar characteristics of melting hail. Part III: Validation of the algorithm for hail size discrimination. J. Appl. Meteor. Climatol., 55, 829-848, https://doi.org/10.1175/JAMCD-15-0203.1.

Park, H. S., A. V. Ryzhkov, D. S. Zrnić, and K.-Y. Kim, 2009: The hydrometeor classification algorithm for the polarimetric WSR-88D: Description and application to an MCS. Wea. Forecasting, 24, 730-748, https://doi.org/10.1175/ 2008WAF2222205.1.

Picca, J. C., and A. V. Ryzhkov, 2012: A dual-wavelength polarimetric analysis of the 16 May 2010 Oklahoma City extreme hailstorm. Mon. Wea. Rev., 140, 1385-1403, https://doi.org/ 10.1175/MWR-D-11-00112.1. 
Rasmussen, R. M., and A. J. Heymsfield, 1987: Melting and shedding of graupel and hail. Part I: Model physics. J. Atmos. Sci., 44, 2754-2763, https://doi.org/10.1175/1520-0469(1987)044<2754: MASOGA $>2.0 . \mathrm{CO} ; 2$.

— - V. Levizzani, and H. R. Pruppacher, 1984: A wind tunnel and theoretical study on the melting behavior of atmospheric ice particles: III. Experiment and theory for spherical ice particles of radius $>500 \mu \mathrm{m}$. J. Atmos. Sci., 41, 381-388, https://doi.org/ 10.1175/1520-0469(1984)041<0381:AWTATS>2.0.CO;2.

Ryzhkov, A. V., 2001: Interpretation of polarimetric radar covariance matrix for meteorological scatterers: Theoretical analysis. J. Atmos. Oceanic Technol., 18,315-328, https://doi.org/10.1175/ 1520-0426(2001)018<0315:IOPRCM > 2.0.CO;2.

- 2007: The impact of beam broadening on the quality of radar polarimetric data. J. Atmos. Oceanic Technol., 24, 729-744, https://doi.org/10.1175/JTECH2003.1.

- and D. S. Zrnić, 1998: Beamwidth effects on the differential phase measurements of rain. J. Atmos. Oceanic Technol., 15, 624-634, https://doi.org/10.1175/1520-0426(1998)015<0624: BEOTDP $>2.0 . \mathrm{CO} ; 2$.

—, M. Pinsky, A. Pokrovsky, and A. Khain, 2011: Polarimetric radar observation operator for a cloud model with spectral microphysics. J. Appl. Meteor. Climatol., 50, 873-894, https:// doi.org/10.1175/2010JAMC2363.1.

— M. R. Kumjian, S. M. Ganson, and A. P. Khain, 2013a: Polarimetric radar characteristics of melting hail. Part I: Theoretical simulations using spectral microphysical modeling. J. Appl. Meteor. Climatol., 52, 2849-2870, https://doi.org/ 10.1175/JAMC-D-13-073.1.

- —_, _ - and P. Zhang, 2013b: Polarimetric radar characteristics of melting hail. Part II: Practical implications. J. Appl.
Meteor. Climatol., 52, 2871-2886, https://doi.org/10.1175/ JAMC-D-13-074.1.

_ , and Coauthors, 2017: Estimation of depolarization ratio using weather radars with simultaneous transmission/reception. J. Appl. Meteor. Climatol., 56, 1797-1816, https://doi.org/10.1175/ JAMC-D-16-0098.1.

Schrom, R. S., 2018: Radar measurements and simulations of ice crystal growth in Arctic mixed-phase clouds. Ph.D. dissertation, Department of Meteorology and Atmospheric Science, The Pennsylvania State University, $180 \mathrm{pp}$.

Snyder, J. C., H. B. Bluestein, G. Zhang, and S. J. Frasier, 2010: Attenuation correction and hydrometeor classification of high-resolution, X-band, dual-polarized mobile radar measurements in severe convective storms. J. Atmos. Oceanic Technol., 27, 1979-2001, https://doi.org/10.1175/ 2010JTECHA1356.1.

Yurkin, M. A., and A. G. Hoekstra, 2011: The discrete-dipole approximation code ADDA: Capabilities and known limitations. J. Quant. Spectrosc. Radiat. Transfer, 112, 2234-2247, https://doi.org/10.1016/j.jqsrt.2011.01.031.

Zeng, Z., S. E. Yuter, R. A. Houze, and D. E. Kingsmill, 2001: Microphysics of the rapid development of heavy convective precipitation. Mon. Wea. Rev., 129, 1882-1904, https://doi.org/10.1175/1520-0493(2001)129<1882:MOTRDO> 2.0.CO;2.

Zrnić, D. S., N. Balakrishnan, and M. Sachidanandan, 1988: Processing and interpretation of alternately polarised weather radar echoes. Int. Geoscience and Remote Sensing Symposium, Remote Sensing: Moving Toward the 21st Century, Edinburgh, United Kingdom, IEEE, 243-245, https://doi.org/10.1109/ IGARSS.1988.570103. 